\title{
Polyphenols as Prebiotics in the Management of High-Fat Diet-Induced Obesity: A Systematic Review of Animal Studies
}

\author{
Mohanambal Moorthy ${ }^{1,2}$, Usha Sundralingam ${ }^{3}(\mathbb{D})$ and Uma D. Palanisamy ${ }^{1,2, *(D)}$ \\ 1 Jeffrey Cheah School of Medicine and Health Sciences, Monash University Malaysia, Jalan Lagoon Selatan, \\ Bandar Sunway 47500, Selangor, Malaysia; Mohanambal.Moorthy@monash.edu \\ 2 Tropical Medicine and Biology Platform, School of Science, Monash University, \\ Bandar Sunway 47500, Selangor, Malaysia \\ 3 School of Pharmacy, Monash University Malaysia, Jalan Lagoon Selatan, \\ Bandar Sunway 47500, Selangor, Malaysia; Usha.Sundralingam@monash.edu \\ * Correspondence: umadevi.palanisamy@monash.edu; Tel.: +60-35514-5840 or +60-1238-09-092
}

Citation: Moorthy, M.;

Sundralingam, U.; Palanisamy, U.D. Polyphenols as Prebiotics in the

Management of High-Fat Diet-Induced Obesity: A Systematic Review of Animal Studies. Foods 2021 10, 299. https://doi.org/10.3390/ foods10020299

Academic Editor: Charis

M. Galanakis

Received: 29 December 2020

Accepted: 25 January 2021

Published: 2 February 2021

Publisher's Note: MDPI stays neutral with regard to jurisdictional claims in published maps and institutional affiliations.

Copyright: (c) 2021 by the authors. Licensee MDPI, Basel, Switzerland. This article is an open access article distributed under the terms and conditions of the Creative Commons Attribution (CC BY) license (https:// creativecommons.org/licenses/by/ $4.0 /)$.
Abstract: Obesity is a disease growing at an alarming rate and numerous preclinical studies have proven the role of polyphenols in managing this disease. This systematic review explores the prebiotic effect of polyphenols in the management of obesity among animals fed on a high-fat diet. A literature search was carried out in PubMed, Scopus, CINAHL, Web of Science, and Embase databases following the PRISMA guidelines. Forty-four studies reported a significant reduction in obesity-related parameters. Most notably, $83 \%$ of the studies showed a decrease in either body weight/visceral adiposity/plasma triacylglyceride. Furthermore, 42 studies reported a significant improvement in gut microbiota (GM), significantly affecting the genera Akkermansia, Bacteroides, Blautia, Roseburia, Bifidobacteria, Lactobacillus, Alistipes, and Desulfovibrio. Polyphenols' anti-obesity, anti-hyperglycaemic, and anti-inflammatory properties were associated with their ability to modulate GM. This review supports the notion of polyphenols as effective prebiotics in ameliorating HFDinduced metabolic derangements in animal models.

Keywords: polyphenols; prebiotics; gut microbiota; obesity; anti-hyperglycaemic; anti-inflammatory

\section{Introduction}

Obesity is a public health concern as it is a risk factor for life-threatening diseases such as type II diabetes [1], cardiovascular diseases [2], certain cancers [1], kidney diseases [3], and neurodegenerative diseases [4]. In the year 2017, overweight and obesity caused over four million deaths, with more than 140 million 'global deaths and disability-adjusted life years' [5]. The interplay between genetic-epigenetic-environmental factors is often linked to obesity [6,7], and of late, the role of gut microbes (GM) in the development of obesity has been extensively explored [8-10].

The GM-obesity relationship was first highlighted by Bäckhed et al. [11] using germfree mice, whereby conventionalisation of such mice with a normal microbiota increased body fat by $60 \%$ and elicited insulin resistance (IR) within 14 days. This finding was supported by Turnbaugh et al. [12], where the administration of microbes of obese or lean mice into germ-free mice, saw higher adiposity in the former. GM's role was further reinforced when germ-free mice were protected against a high-fat diet (HFD)-induced obesity [13]. Collectively these studies proved the role of GM in energy harvest and obesity. Consequently, subsequent studies proposed dietary intervention for GM's modulation, and substantial work involving probiotics, prebiotics or synbiotics in the management of obesity had emerged.

Polyphenols (PP) are secondary metabolites found abundantly in plants, fruits and nuts [14]. A growing body of research indicates that PP possess various biological activities 
ranging from anti-oxidant [15,16], anti-inflammatory [15], hepatoprotective [16], antihyperglycaemic [17,18], anti-cancer [19], neuroprotective [20,21], and cardioprotective [22]. Therefore, PP are even considered an excellent alternative to harmful food preservatives and additives [23-25] as well as nutraceutical agents [26]. Despite the vast health benefits, PP were shown to be poorly bioavailable and their interaction with gut microbiota was highlighted as the main factor for their bioactivities [27,28]. The PP-GM interaction in return is described to affect the intestinal ecology and influence host health [28]. Based on the International Scientific Association for Probiotics and Prebiotics consensus document, prebiotics is described as "a substrate that is selectively utilised by host microorganisms and conferring a health benefit" [29]. Therefore, these phytochemicals are now beginning to emerge as the new prebiotics [30].

Growing preclinical studies have confirmed the anti-obesity properties of PP to be dependent on their interaction with GM. Nevertheless, a comprehensive review is still lacking. We undertook a systematic review to investigate the effect of PP administration on obesity-related parameters, markers associated with inflammation, and gut microbiota in HFD-fed animal models. This study is essential to elucidate if PP's effectiveness in ameliorating metabolic derangements is indeed associated with GM modulation.

\section{Methods}

The protocol of this systematic review was registered with PROSPERO (www.crd. yor.ac.uk/PROSPERO; CRD42020153321). This review's reporting followed the Preferred Reporting Items for Systematic Reviews and Meta-Analysis (PRISMA) [31].

\subsection{Search Strategy}

MM and USL undertook a comprehensive literature search in the following databases; CINAHL, Excerpta Medica Database (Embase), Scopus, PubMed, and Web of Science. A published search term relating to PP and GM were used [30] (Table 1) (Figure S1-search strategy (PubMed)). All the acquired bibliographic records were exported to Endnote X8 (Thomson Reuters, New York, NY, USA), following which duplicates were removed. We also carried out a manual search of bibliographies of reviews and included studies to identify relevant articles.

\subsection{Eligibility Criteria}

Studies that met the following criteria were included in this review: (1) In vivo studies of any species of animal which were subjected to HFD; (2) Treatment and control animal of the same species; (3) Intervention with either pure phenolic compounds or its extracts or both; (4) Studies that measured the phenolic contents if PP rich extracts were used; (5) Studies that evaluated gut microbiota composition via next-generation sequencing methods; (6) Studies reporting on obesity-related parameters (body weight, adiposity, fasting blood glucose, fasting insulin, HOMA-IR), markers associated with inflammation (adipocytokines, endotoxins); (7) Papers published in the English language. The exclusion criteria were: (1) Combination of PP with other compounds/bacterial species. e.g., PP with fibre, PP with probiotic; (2) HFD in-combined with any diabetogens such as streptozotocin; (3) Faecal transplantation from polyphenol treated animals; (4) Fermented PP; (5) Conference abstracts; (6) Reviews/meta-analysis.

\subsection{Data Extraction}

Two reviewers, MM and USL, independently screened the titles and abstracts based on the selection criteria before evaluating the full text. Disagreements or discrepancies were discussed with a third reviewer (UDP) or resolved by consensus. The following information extracted from the full text was included in this review; the name of the first author, publication year, animal: Species, sex, age, and number animals per group, intervention: Prevention/treatment mode, percentage of fat in HFD, duration of intervention, type of PP, dose, and mode of administration, outcomes: Obesity-related parameters [body 
weight, the weight of visceral adipose tissue (VAT), the weight of subcutaneous adipose tissue (SAT), glucose, insulin, lipid profile], markers of inflammation (endotoxin, and adipocytokines), and gut microbiota modulation: Alpha-diversity, beta-diversity (based on PCoA/PCA/NMDS plots), modulation of phyla, family or genus, Firmicutes:Bacteroidetes ratio.

Table 1. Search terms.

\begin{tabular}{cc}
\hline Polyphenol & Gut Microbiota \\
\hline Dietary polyphenol & \\
Polyphenol & \\
Flavonoid & \\
Fruit & \\
Vegetable & \\
Plant extract & \\
Herbal drug & \\
Medicinal plant & \\
Antioxidant & \\
Anthocyanin & Microbiota \\
Chalcones & Gut microbiota \\
Catechin & Colonic microbiota \\
Flavanone & Gastrointestinal microbiota \\
Proanthocyanidin & Intestinal microbiota \\
Ellagitannin & Gut organism \\
Functional food & Microbial consortia \\
Green tea & Gut bacterium \\
Puerh tea & Gut flora \\
Cocoa & Gastrointestinal flora \\
Chocolate & Intestinal flora \\
Myo-inositol & \\
Soy isoflavone & \\
Blueberries & Berries \\
Grape & \\
Quercetin & \\
Citrus & \\
Red wine & \\
Resveratrol & \\
Natural s-equol & \\
& \\
\hline
\end{tabular}

\subsection{Quality Assessment}

The methodological quality of the included studies was assessed independently by MM and USL using the SYRCLE's risk of bias tool [32] and modified Gold Standard Publication Checklist (GSPC) [33,34]. Any disagreement or discrepancies were resolved by consensus. The SYRCLE's checklist includes sequence generation, baseline characteristics, allocation concealment, random housing, blinding, random outcome assessment, blinding (outcome), incomplete outcome data and also other sources of bias. Each study was graded as having a low risk, high risk or unclear risk for each domain. GSPC quantitatively assesses various criteria, but only specific criteria were used in this review (Table S1) [34]. Each criterion was given ' 0 ' if not addressed in the article or ' 1 ' if addressed, and the total score assigned was 19.

\section{Results}

Our database search resulted in 17,176 articles. Following duplicate removal and abstract screening, 350 full-text articles were screened. Only 45 articles that met our inclusion criteria were included in this review, as outlined in Figure 1. Table 2 summarises the characteristics of the included studies. 

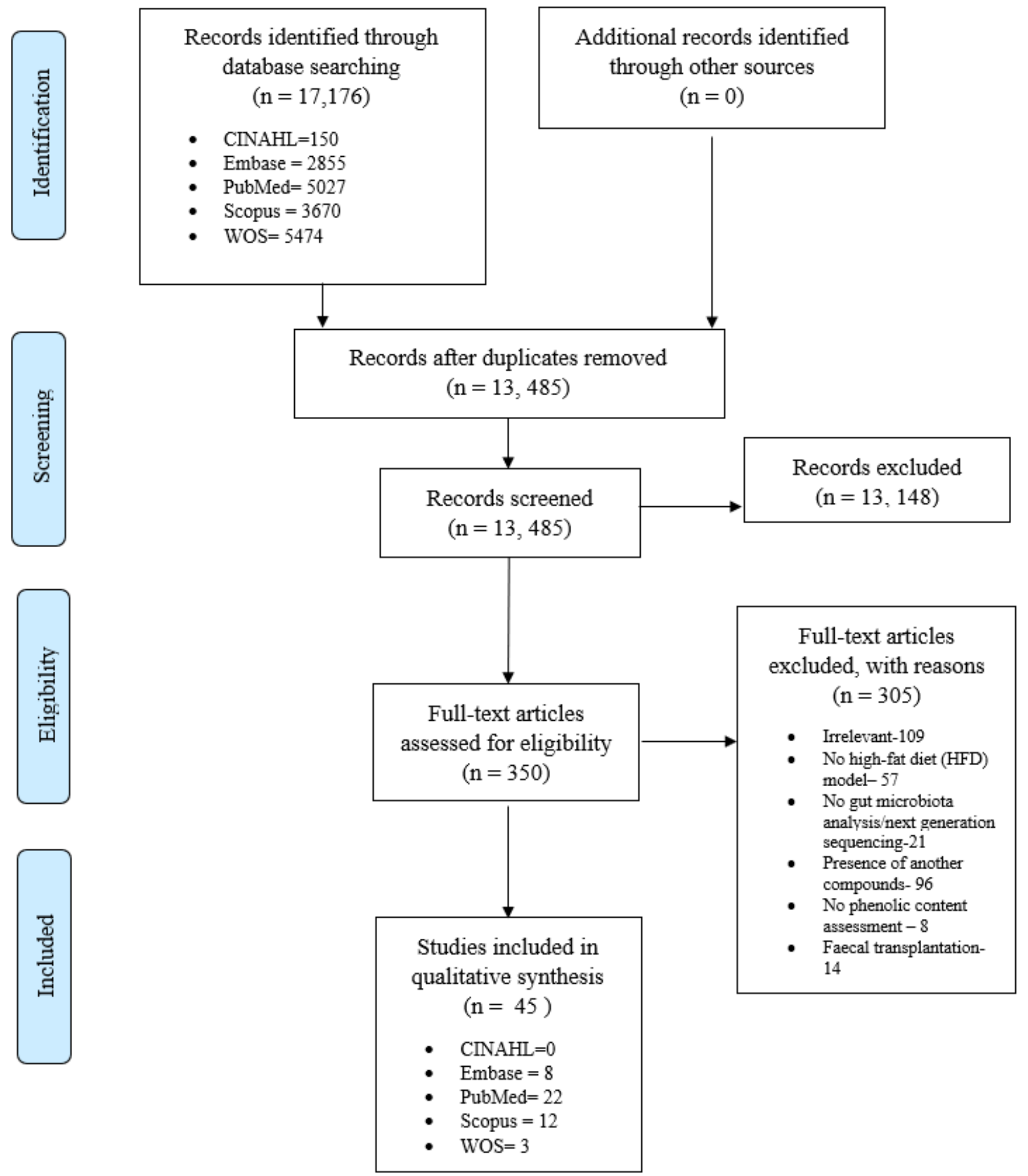

Figure 1. PRISMA Flow-chart. 
Table 2. Characteristics of reviewed animal studies.

\begin{tabular}{|c|c|c|c|c|c|c|c|c|c|c|c|c|c|c|c|c|c|c|}
\hline No. & $\begin{array}{l}\text { Author, } \mathrm{Y}_{\mathbf{r}} \\
\text { [Reference] }\end{array}$ & $\begin{array}{c}\text { Species, } \\
\text { Sex, Age } \\
\text { (w)/ } \\
\text { Weight (g) }\end{array}$ & $\begin{array}{l}\text { Number of } \\
\text { Animals, } n / \\
\text { Groups (Grp) }\end{array}$ & $\begin{array}{l}\text { Prevention } \\
(p) / / \text { Treatment } \\
\text { (T) }\end{array}$ & $\begin{array}{l}\text { Duration of } \\
\text { Intervention } \\
\text { (d/wss/mthss) }\end{array}$ & $\begin{array}{l}\text { HFD } \\
(\% \text { Fat })\end{array}$ & Dosage & MOA & $\begin{array}{c}\text { Sample/Method/ } \\
\text { Hypervariable Region/GM } \\
\text { Composition }\end{array}$ & $\begin{array}{l}\text { Energy/Food } \\
\text { Intake }\end{array}$ & Weight & $\begin{array}{l}\text { VAT/ } \\
\text { SAT }\end{array}$ & $\begin{array}{c}\text { Glucose } \\
\text { (FG/GTT/ITT) }\end{array}$ & Hormones & HOMA-IR & Adipocytokine & Lipid Profile & Endotoxins \\
\hline & \multicolumn{18}{|c|}{ PURE COMPOUNDS-MICE } \\
\hline 1 & $\begin{array}{l}\text { Lópeze et al., } \\
2018[35]\end{array}$ & $\begin{array}{c}\text { C57BL6 } 6 \\
\text { mice, } \mathrm{M}, 9 \mathrm{w}\end{array}$ & $\begin{array}{l}\text { 8/grp ND HFD } \\
\text { + genistein(G) }\end{array}$ & $p$ & $24 \mathrm{w}$ & $\mathrm{NA}$ & $\begin{array}{l}\text { Genistein } \\
-0.2 \%\end{array}$ & $\begin{array}{l}\text { Within } \\
\text { diet }\end{array}$ & 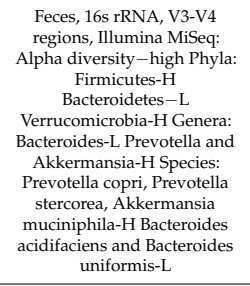 & SL & SL & SAT:SL & $\begin{array}{c}\text { FG-NS } \\
\text { IpGTT(AUC)-SL }\end{array}$ & & & & $\begin{array}{l}\text { TC-NS } \\
\text { TAG-SL } \\
\text { LDL-NS }\end{array}$ & LPS-SL \\
\hline 2 & $\begin{array}{l}\text { X. Guo et al., } \\
2018 \text { [36] }\end{array}$ & $\begin{array}{c}\mathrm{C} 57 \mathrm{BL} / 6 \mathrm{~J} \\
\text { mice, } \mathrm{M}, 4 \mathrm{w}\end{array}$ & $\begin{array}{l}\text { 12/grp ND } \\
\text { HFD HFD + R } \\
\text { HFD + R+ } \\
\text { Innlin * only } \\
\text { results for rutin } \\
\text { reported in this } \\
\text { review }\end{array}$ & $p$ & $20 \mathrm{w}$ & $60 \%$ & $\begin{array}{c}\text { Rutin (R)-6.4 } \\
\text { mg/g diet }\end{array}$ & $\begin{array}{c}\text { Within } \\
\text { diet }\end{array}$ & 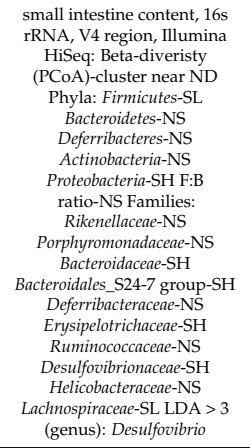 & NS & SL & & & & & $\begin{array}{l}\text { Leptin-SL } \\
\text { II-6-SL } \\
\text { TIFa-SL } \\
\text { IFN-S-SL } \\
\text { IL-2-SLL IL4-SL }\end{array}$ & $\begin{array}{l}\text { TC-NS } \\
\text { TAG-NS }\end{array}$ & LPS-SL \\
\hline 3 & $\begin{array}{l}\text { Masumoto } \\
\text { et al, 2016 } \\
\text { [37] }\end{array}$ & $\begin{array}{c}\text { C57BL/6Jmice, } \\
\mathrm{M}, \\
9 \mathrm{w}\end{array}$ & $\begin{array}{l}\text { 10/grp ND } \\
\text { HFHS HHS + } \\
\text { OP HFHS + PP }\end{array}$ & $\mathrm{NA}$ & $20 \mathrm{w}$ & $\mathrm{NA}$ & $\begin{array}{l}\text { Oligomeric } \\
\text { procyani- } \\
\text { dins } \\
\text { (OP)-NA } \\
\text { Polyeric } \\
\text { procyani- } \\
\text { prins } \\
\text { (PPs)-0.5\% }\end{array}$ & $\mathrm{NA}$ & 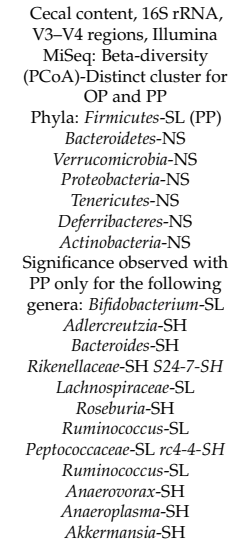 & $\mathrm{OP}, \mathrm{PP}-\mathrm{NS}$ & $\begin{array}{l}\text { OP-NS, } \\
\text { PP-SL }\end{array}$ & $\begin{array}{l}\text { VAT: OP, } \\
\text { PP-SLAT: } \\
\text { OP, PP-SL }\end{array}$ & OP, PP-SL & & & $\begin{array}{l}\text { TNFa: PP-SL } \\
\text { IL-6:PP-SL }\end{array}$ & $\begin{array}{l}\text { TC: OP, PP- } \\
\text { SLTAGGOP, } \\
\text { PP-SL }\end{array}$ & LPS:PP-SL \\
\hline
\end{tabular}


Table 2. Cont.

\begin{tabular}{|c|c|c|c|c|c|c|c|c|c|c|c|c|c|c|c|c|c|c|}
\hline No. & $\begin{array}{l}\text { Author, } \mathrm{Yr}_{\mathrm{r}} \\
\text { [Reference] }\end{array}$ & $\begin{array}{c}\text { Species, } \\
\text { Sex, Age } \\
\text { (w)/ } \\
\text { Weight (g) }\end{array}$ & $\begin{array}{l}\text { Number of } \\
\text { Animals, } n \\
\text { Groups (Grp) }\end{array}$ & $\begin{array}{l}\text { Prevention } \\
(p) / \text { /reatment } \\
\text { (T) }\end{array}$ & $\begin{array}{l}\text { Duration of } \\
\text { Intervention } \\
\text { (d/wks/mths) }\end{array}$ & $\begin{array}{l}\mathrm{HFD} \\
(\% \mathrm{Fat})\end{array}$ & Dosage & MOA & $\begin{array}{c}\text { Sample/Method/ } \\
\text { Hypervariable Region/GM } \\
\text { Composition }\end{array}$ & $\begin{array}{l}\text { Energy/Food } \\
\text { Intake }\end{array}$ & Weight & $\begin{array}{l}\mathrm{VAT} / \\
\text { SAT }\end{array}$ & $\begin{array}{c}\text { Glucose } \\
\text { (FG/GTT/ITT) }\end{array}$ & Hormones & HOMA-IR & Adipocytokine & Lipid Profile & Endotoxins \\
\hline 4 & $\begin{array}{l}\text { Tung et al., } \\
2016[38]\end{array}$ & $\begin{array}{l}\mathrm{C57BL} / 6 \\
\text { mice, } \mathrm{M}, \\
5 \mathrm{w}\end{array}$ & $\begin{array}{c}\text { 8/grp ND HFD } \\
\text { HFD + } 0.1 \% \mathrm{R} \\
\mathrm{HF}+0.1 \% \text { Pic } \\
\text { (LP)HFD+ } \\
0.25 \% \operatorname{Pic}(\mathrm{HP})\end{array}$ & $p$ & $18 \mathrm{w}$ & $45 \%$ & 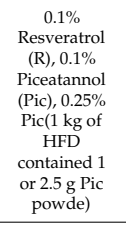 & $\begin{array}{l}\text { Withi } \\
n \text { diet }\end{array}$ & 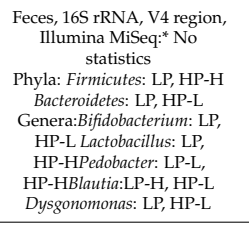 & $\begin{array}{l}\text { R, LP, } \\
\text { HP-NS }\end{array}$ & $\begin{array}{c}\text { LP, } \\
\text { HP-SL }\end{array}$ & 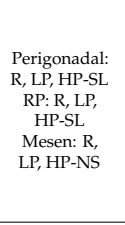 & FG:LP, HP-SL & & & & $\begin{array}{l}\text { TC: LP, } \\
\text { HP.SL } \\
\text { TAG:LP, HP, } \\
\text { R-NS } \\
\text { LDL:HP-SL } \\
\text { HDL: } \\
\text { HPP }-S L\end{array}$ & \\
\hline 5 & $\begin{array}{l}\text { Porras et al., } \\
2017[39]\end{array}$ & $\begin{array}{l}\mathrm{C57BL/6J} \\
\text { mice, M, } \\
7 \mathrm{w}\end{array}$ & $\begin{array}{l}\text { 10/grp:NDND } \\
\text { + QHFDHFD + } \\
\text { Q }\end{array}$ & $p$ & $16 \mathrm{w}$ & $60 \%$ & 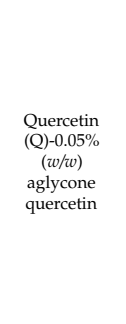 & $\begin{array}{l}\text { Within } \\
\text { diet }\end{array}$ & 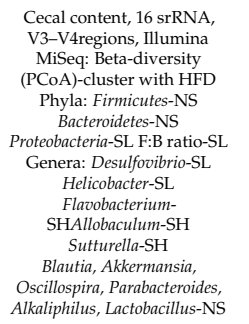 & NS & SL & Epid-SL & FG-SL & FI-SL & SL & IL-6-SL & $\begin{array}{l}\text { Plasma:TAG- } \\
\text { SLLiver:TAG, } \\
\text { FFA-SL }\end{array}$ & LPS-SL \\
\hline 6 & $\begin{array}{l}\text { Brandt etal., } \\
2018 \text { [40]] }\end{array}$ & $\begin{array}{l}\text { C57BL/ } 6 \mathrm{~N} \\
\text { with loxP } \\
\text { insertions in } \\
\text { the Pparcla } \\
\text { gene, M, } \\
8-10 \mathrm{w}\end{array}$ & $\begin{array}{l}n=\text { NANDHFD- } \\
\text { HFD + R HFD + } \\
\text { exercise (Ex) } \\
\text { *only results } \\
\text { for resveratrol } \\
\text { reported in this } \\
\text { review }\end{array}$ & $p$ & $16 \mathrm{w}$ & $60 \%$ & $\begin{array}{l}\text { Resveratrol } \\
\text { (R)-4g//kg } \\
\text { HFD }\end{array}$ & $\begin{array}{l}\text { Within } \\
\text { diet }\end{array}$ & 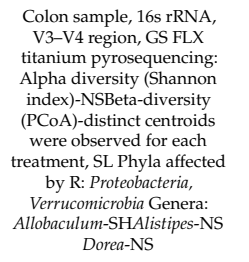 & SL & SL & $\begin{array}{l}\text { VAT: NSSAT: } \\
\text { NS }\end{array}$ & & & & $\begin{array}{l}\text { Serum Amyloid } \\
\text { A-NS }\end{array}$ & & \\
\hline 7 & 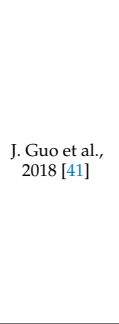 & $\begin{array}{l}\mathrm{C} 57 \mathrm{BL} / 6 \mathrm{~J} \\
\text { mice, M, } \\
3 \mathrm{w}\end{array}$ & $\begin{array}{l}\text { 7-8/grp ND } \\
\text { HFDHFD + V }\end{array}$ & $p$ & $14 \mathrm{w}$ & $60 \%$ & $0.1 \%$ vanillin & $\begin{array}{l}\text { Within } \\
\text { diet }\end{array}$ & 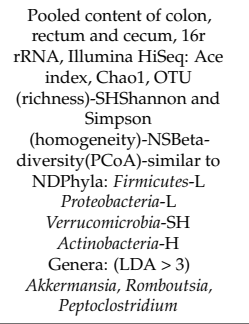 & SH & SL & $\begin{array}{c}\text { Epid-SL } \\
\text { Inguinal-SL }\end{array}$ & $\begin{array}{c}\text { FG-SL } \\
\text { OGTT(AUC)-SL } \\
\text { ITT(AUC)-SL }\end{array}$ & & & $\begin{array}{l}\text { TNFa-SL } \\
\text { IL-6-SL }\end{array}$ & $\begin{array}{l}\text { TC-SL } \\
\text { TAG-SL } \\
\text { LDL-SL } \\
\text { HDL-NS }\end{array}$ & LPS-SL \\
\hline 8 & $\begin{array}{l}\text { Cremonini } \\
\text { et al, } 2019 \\
{[42], \text { Daveri }} \\
\text { et al, 2018 } \\
{[43]} \\
{[43]}\end{array}$ & $\begin{array}{l}\text { C57BL/6J } \\
\text { mice, M, } \\
(20-25 \mathrm{~g})\end{array}$ & $\begin{array}{l}\text { 10/grp NDND } \\
+ \text { AC HFDHFD } \\
+ \text { +AC }\end{array}$ & p & $14 \mathrm{w}$ & $60 \%$ & $\begin{array}{c}\text { Anthocyanins } \\
\text { (AC)- } \\
40 \mathrm{mg} / \mathrm{kg}\end{array}$ & $\begin{array}{l}\text { Within } \\
\text { diet }\end{array}$ & $\begin{array}{l}\text { Cecal content, 16s rRNA, V4 } \\
\text { region, Ilumina Miseq } \\
\text { Beta-diversity(NNDDSS-closer } \\
\text { to ND } \\
\text { F:B ratio-SL } \\
\text { Akkermansia-SH }\end{array}$ & SL & SL & $\begin{array}{l}\text { VAT: SL } \\
\text { SAT: SL }\end{array}$ & $\begin{array}{c}\text { FG-SL } \\
\text { OGTT(AUC)-SL } \\
\text { ITT(AUC)-SL }\end{array}$ & $\begin{array}{c}\text { FI-SL } \\
\text { GIP, } \\
\text { GLP-1-SL } \\
\text { GLP-2- } \\
\text { SH }\end{array}$ & & $\begin{array}{l}\text { Leptin-SL } \\
\text { Adiponection- } \\
\text { NS }\end{array}$ & $\begin{array}{c}\text { Plasma: } \\
\text { TC-SL } \\
\text { TAG-sL } \\
\text { Liver.TAG- } \\
\text { SL }\end{array}$ & $\begin{array}{l}\text { Endotoxin- } \\
\text { SL } \\
\text { FITC- } \\
\text { dextran-SL }\end{array}$ \\
\hline
\end{tabular}


Table 2. Cont.

\begin{tabular}{|c|c|c|c|c|c|c|c|c|c|c|c|c|c|c|c|c|c|c|}
\hline No. & $\begin{array}{l}\text { Author, } \mathrm{Yr}_{\mathrm{r}} \\
\text { [Reference] }\end{array}$ & $\begin{array}{l}\text { Species, } \\
\text { Sex, Age } \\
\text { (w)/ } \\
\text { Weight (g) }\end{array}$ & $\begin{array}{l}\text { Number of } \\
\text { Animals, } n / \\
\text { Groups (Grp) }\end{array}$ & $\begin{array}{l}\text { Prevention } \\
(p) / \text { Treatment } \\
\text { (T) }\end{array}$ & $\begin{array}{l}\text { Duration of } \\
\text { Intervention } \\
\text { (d/wks/mths) }\end{array}$ & $\begin{array}{l}\mathrm{HFD} \\
(\% \mathrm{Fat})\end{array}$ & Dosage & MOA & $\begin{array}{c}\text { Sample/Method/ } \\
\text { Hypervariable Region/GM } \\
\text { Composition }\end{array}$ & $\begin{array}{l}\text { Energy/Food } \\
\text { Intake }\end{array}$ & Weight & $\begin{array}{l}\mathrm{VAT} / \\
\text { SAT }\end{array}$ & $\begin{array}{c}\text { Glucose } \\
\text { (FG/GTT/ITT) }\end{array}$ & Hormones & HOMA-IR & Adipocytokine & Lipid Profile & Endotoxins \\
\hline 9 & 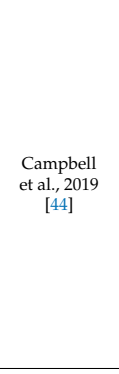 & $\begin{array}{l}\mathrm{C} \text { C57BL/6J } \\
\text { mice, M, } \\
4 \mathrm{w}\end{array}$ & $\begin{array}{l}\text { 12/grp ND } \\
\text { HFD HFD + L } \\
\text { HFD + M } \\
\text { HFD + H }\end{array}$ & $\mathrm{T}$ & $12 \mathrm{w}$ & $35 \%$ & $\begin{array}{l}\mathrm{HFD}+\mathrm{L}- \\
50 \mathrm{mg} / \mathrm{kg} / \text { day } \\
\mathrm{HFD}+\mathrm{M}- \\
75 \mathrm{mg} / \mathrm{kg} / \text { day } \\
\mathrm{HFD}+\mathrm{H}- \\
100 \\
\mathrm{mg} / \mathrm{kg} / \mathrm{gay} \\
\text { Resveratrol } \\
\text { were } \\
\text { dissolved in } \\
0.4 \mathrm{~mL} \text { of } \\
\text { absolute } \\
\text { ethanol and } \\
\text { added to } \\
\text { and } \\
\text { drink of } \\
\text { dring } \\
\text { water daily } \\
\end{array}$ & $\begin{array}{l}\text { Drinking } \\
\text { water }\end{array}$ & 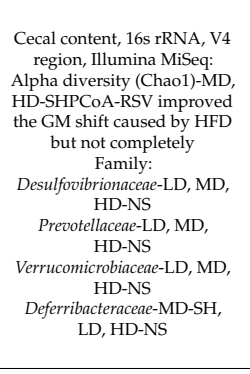 & $\begin{array}{l}\text { LD, MD, } \\
\text { HD-NS }\end{array}$ & $\begin{array}{l}\text { LD-NS } \\
\text { MD-SL } \\
\text { HD-SL }\end{array}$ & $\begin{array}{c}\text { Epid, } \\
\text { perinephric, } \\
\text { mesen:LD- } \\
\text { NS } \\
\text { MD-SL } \\
\text { HD-SL }\end{array}$ & $\begin{array}{l}\text { FG: LD, MD, } \\
\text { HD-SL }\end{array}$ & & & $\begin{array}{l}\text { IL-1: MD, } \\
\text { HD-SLIL-10: } \\
\text { LD, MD, HD-SL } \\
\text { TNFa: MD, } \\
\text { HD-SL }\end{array}$ & $\begin{array}{l}\text { TAG: LD, } \\
\text { MD, HD-SL } \\
\text { HDL:HD- } \\
\text { SH } \\
\text { LDL:LD, } \\
\text { MD, HD-SL }\end{array}$ & $\begin{array}{l}\text { LPS:LD, MD, } \\
\text { HD-SL } \\
\text { LBP: } \\
\text { MD, } \\
\text { HD-SL }\end{array}$ \\
\hline 10 & $\begin{array}{l}\text { Zheng et al., } \\
2018[455]\end{array}$ & $\begin{array}{l}\text { C578L/6J } \\
\text { mice, } \mathrm{M}, 3 \mathrm{w}\end{array}$ & $\begin{array}{l}\text { 6/grp: LFHFD } \\
\text { HFD }+p\end{array}$ & $\mathrm{~T}$ & 12 & $60 \%$ & $\begin{array}{l}\text { Procyanidin(p) } \\
100 \mathrm{mg} / \mathrm{kg}\end{array}$ & $\begin{array}{l}\text { Oral } \\
\text { gav- } \\
\text { age }\end{array}$ & 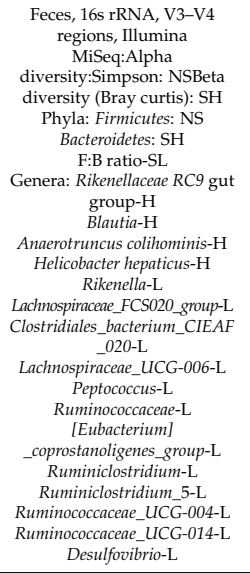 & & SL & VAT: NS & & & & & $\begin{array}{l}\text { TC-SH } \\
\text { TAG-NS } \\
\text { HDL-SH } \\
\text { LDL-NS }\end{array}$ & \\
\hline 11 & $\begin{array}{l}\text { Xie et al., } \\
2018[46]\end{array}$ & $\begin{array}{l}\mathrm{C} 57 \mathrm{BL} / 6 \\
\text { mice, } \mathrm{M}, \\
6 \mathrm{w}\end{array}$ & $\begin{array}{l}\text { 8/grp ND ND + } \\
\text { LD HFD HFD + } \\
\text { LD HFD + HD }\end{array}$ & $p$ & $9 \mathrm{w}$ & $45 \%$ & $\begin{array}{l}\text { kudingcha } \\
\text { dicaf- } \\
\text { feoylquinic } \\
\text { acids Low } \\
\text { does } \\
\text { (LD)-3.3 } \\
\text { mg/mouse } \\
\text { high dose } \\
\text { (mD)-10.0 } \\
\text { mg/mouse }\end{array}$ & $\begin{array}{l}\text { Oral } \\
\text { gav- } \\
\text { age }\end{array}$ & 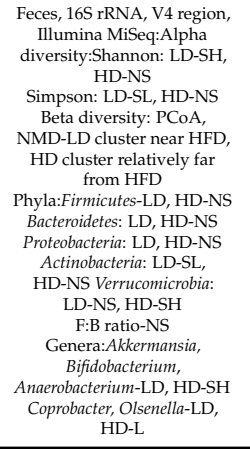 & & NS & $\begin{array}{l}\text { Perirenal: } \\
\text { LD, HD-SL } \\
\text { Epid:HD-SL }\end{array}$ & & & & $\begin{array}{c}\text { CRP:LD, HD-SL } \\
\text { TNFa:LD, } \\
\text { HD-SL } \\
\text { II-6:LD, HD-SL }\end{array}$ & $\begin{array}{l}\text { TC:LD-NS, } \\
\text { HD-SL } \\
\text { TAG:LD, } \\
\text { HD-NS } \\
\text { LDLL LD, } \\
\text { HD-SL } \\
\text { HDL: } \\
\text { LD-NS, } \\
\text { HD-SL }\end{array}$ & $\begin{array}{l}\text { LPS:LD, } \\
\text { HD-SL }\end{array}$ \\
\hline
\end{tabular}


Table 2. Cont.

\begin{tabular}{|c|c|c|c|c|c|c|c|c|c|c|c|c|c|c|c|c|c|c|}
\hline No. & $\begin{array}{l}\text { Author, } \mathrm{Yr}_{\mathrm{r}} \\
\text { [Reference] }\end{array}$ & $\begin{array}{c}\text { Species, } \\
\text { Sex, Age } \\
\text { (w)l } \\
\text { Weight (g) }\end{array}$ & $\begin{array}{l}\text { Number of } \\
\text { Animals, } n \\
\text { Groups (Grp) }\end{array}$ & $\begin{array}{l}\text { Prevention } \\
(p) / \text { Treatment } \\
\text { (T) }\end{array}$ & $\begin{array}{l}\text { Duration of } \\
\text { Intervention } \\
\text { (d/wks/mths) }\end{array}$ & $\underset{(\% \text { Fat })}{\mathrm{HFD}}$ & Dosage & MOA & $\begin{array}{c}\text { Sample/Method/ } \\
\text { Hypervariable Region/GM } \\
\text { Composition }\end{array}$ & $\begin{array}{l}\text { Energy/Food } \\
\text { Intake }\end{array}$ & Weight & $\begin{array}{l}\text { VAT/ } \\
\text { SAT }\end{array}$ & $\begin{array}{c}\text { Glucose } \\
\text { (FG/GTT/ITT) }\end{array}$ & Hormones & HOMA-IR & Adipocytokine & Lipid Profile & Endotoxins \\
\hline 12 & $\begin{array}{l}\text { Sung et al., } \\
2017[47]\end{array}$ & $\begin{array}{l}\mathrm{C} 57 \mathrm{BL} / 6 \mathrm{~N} \\
\text { mice, } \mathrm{M}, \\
8 \mathrm{w}\end{array}$ & $\begin{array}{c}\text { 10/grp NDND } \\
+ \text { RHFHSHFHs } \\
+\quad \text { R }\end{array}$ & $p$ & $8 \mathrm{w}$ & $45 \%$ & $\begin{array}{c}\text { Resveratrol } \\
(\mathrm{R})-0.4 \%\end{array}$ & $\begin{array}{c}\text { Within } \\
\text { diet }\end{array}$ & 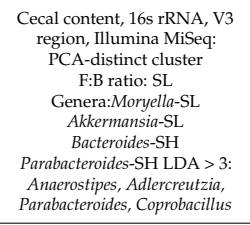 & & NS & $\begin{array}{l}\text { Total body } \\
\text { fat: SL }\end{array}$ & $\begin{array}{l}\text { OGTT (AUC): } \\
\text { SL }\end{array}$ & & & & & \\
\hline 13 & $\begin{array}{l}\text { Zhuoqun } \\
\text { Liet at al., } \\
2019 \text { [48] }\end{array}$ & $\begin{array}{c}\mathrm{C} 57 \mathrm{BL} / 6 \mathrm{~J} \\
\text { mice, M, } \\
3 \mathrm{w}\end{array}$ & $\begin{array}{c}\text { 7/grp ND HFD } \\
\text { HFD + HTHFD } \\
\text { + Fecal } \\
\text { transplantation } \\
\text { (FT) } \\
\text { *only results } \\
\text { for HT reported } \\
\text { in this review }\end{array}$ & $p$ & $8 w$ & $45 \%$ & $\begin{array}{l}\text { Hydroxytyrosol } \\
\text { (HT) -50 } \\
\text { mg/kg/day }\end{array}$ & $\begin{array}{l}\text { Oral } \\
\text { gav- } \\
\text { age }\end{array}$ & 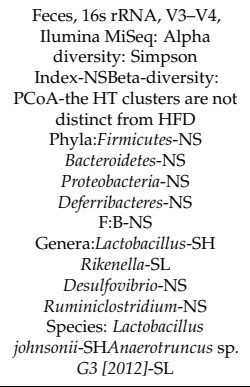 & NS & NS & $\begin{array}{l}\text { RP-SL } \\
\text { Epid-SL } \\
\text { SAT: NS }\end{array}$ & $\begin{array}{c}\text { FG-SL } \\
\text { OGTT(AUC)- } \\
\text { NS } \\
\text { ITT(AUC)-NS }\end{array}$ & FI-SL & SL & & & LPS-SL \\
\hline 14 & $\begin{array}{l}\text { Ushiroda } \\
\text { et al., 2019 } \\
{[49]}\end{array}$ & $\begin{array}{l}\mathrm{C} 57 \mathrm{BL} / 6 \mathrm{~N} \\
\text { mice, } \mathrm{M}, \\
5 \mathrm{w}\end{array}$ & $\begin{array}{c}\text { 8/grp } \\
\text { NDDFPHFD + } \\
\text { EGCG }\end{array}$ & $p$ & $8 \mathrm{w}$ & $32 \%$ & $\begin{array}{l}\text { Epigallocatechin } \\
\text { gallate } \\
\text { (EGCG)- } \\
0.32 \% \\
\text { within diet }\end{array}$ & $\begin{array}{c}\text { Within } \\
\text { diet }\end{array}$ & 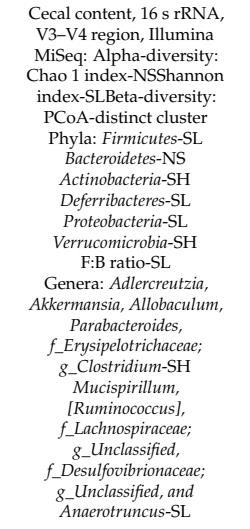 & NS & NS & Epid-NS & & & & & $\begin{array}{l}\text { Serum:TC, } \\
\text { TAG, HDL, } \\
\text { LDL, } \\
\text { NEFA-NS } \\
\text { Liver:TAG- } \\
\text { SL }\end{array}$ & \\
\hline
\end{tabular}


Table 2. Cont.

\begin{tabular}{|c|c|c|c|c|c|c|c|c|c|c|c|c|c|c|c|c|c|c|}
\hline No. & $\begin{array}{l}\text { Author, } \mathrm{r}_{\mathbf{r}} \\
\text { [Referencel }\end{array}$ & $\begin{array}{c}\text { Species, } \\
\text { Sex, Age } \\
\text { (w)/ } \\
\text { Weight (g) }\end{array}$ & $\begin{array}{l}\text { Number of } \\
\text { Animals, } n / \\
\text { Groups (Grp) }\end{array}$ & $\begin{array}{l}\text { Prevention } \\
(p) / / \text { Treatment } \\
\text { (T) }\end{array}$ & $\begin{array}{l}\text { Duration of } \\
\text { Intervention } \\
\text { (d/ws/mths) }\end{array}$ & $\begin{array}{c}\text { HFD } \\
(\% \text { Fat })\end{array}$ & Dosage & MOA & $\begin{array}{c}\text { Sample/Method/ } \\
\text { Hypervariable Region/GM } \\
\text { Composition }\end{array}$ & $\begin{array}{c}\text { Energy/Food } \\
\text { Intake }\end{array}$ & Weight & $\begin{array}{l}\text { VAT/ } \\
\text { SAT }\end{array}$ & $\begin{array}{c}\text { Glucose } \\
\text { (FG/GTT/TT) }\end{array}$ & Hormones & НОМА-IR & Adipocytokine & Lipid Profile & Endotoxins \\
\hline 15 & $\begin{array}{l}\text { Sheng et al,, } \\
2018[50]\end{array}$ & $\begin{array}{c}\text { C57BL/6 } \\
\text { wild-type } \\
\text { (WT) mice, } \\
\text { M, } \\
3 \text { w }\end{array}$ & $\begin{array}{c}\text { ND WDWD + } \\
\text { EGCG WD + } \\
\text { vancomycin + } \\
\text { polymyxin B + } \\
\text { AbxDD + } \\
\text { Akkermansia } \\
\text { muciniphila } \\
\text { supplementa- } \\
\text { tion *only } \\
\text { results for } \\
\text { EGCG reported } \\
\text { in this review }\end{array}$ & $\mathrm{T}$ & $8 \mathrm{w}$ & $21 \%$ & $\begin{array}{l}\text { Epigallocatechin } \\
\text { gallate } \\
\text { (EGCG) } \\
-100 \\
\text { ug/d/gram } \\
\text { body weight }\end{array}$ & $\begin{array}{l}\text { Oral } \\
\text { gav- } \\
\text { age }\end{array}$ & 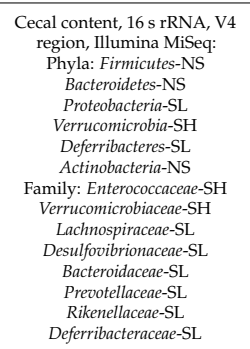 & SH & SL & SL & $\begin{array}{l}\text { FG-NSITT } \\
\text { (AUC)-SL }\end{array}$ & $\begin{array}{l}\text { PYY- } \\
\text { SHGLP- } \\
1 \text {-SH }\end{array}$ & & & $\begin{array}{c}\text { TC-SL } \\
\text { TAG-SL }\end{array}$ & LPS-SL \\
\hline 16 & $\begin{array}{l}\text { W. Liu et al,, } \\
2017 \text { [51] }\end{array}$ & $\begin{array}{l}\mathrm{C} 57 \mathrm{BL} / 6 \\
\text { mice, } \mathrm{M}, \\
8 \mathrm{w}\end{array}$ & $\begin{array}{c}\text { 10-12/grp ND } \\
\text { HFDHFD + } \\
\text { GSPE HFD + } \\
\text { antibiotics + } \\
\text { GSPEHFD+ } \\
\text { antibiotics* } \\
\text { only results for } \\
\text { GSPE reported } \\
\text { in this review }\end{array}$ & $p$ & $7 \mathrm{w}$ & $60 \%$ & $\begin{array}{c}300 \mathrm{mg} / \mathrm{kg} \\
\text { body weight } \\
\text { grape seed } \\
\text { proantho- } \\
\text { cyanidin } \\
\text { extract } \\
\text { (GSPE) }\end{array}$ & $\begin{array}{l}\text { Oral } \\
\text { gav- } \\
\text { age }\end{array}$ & 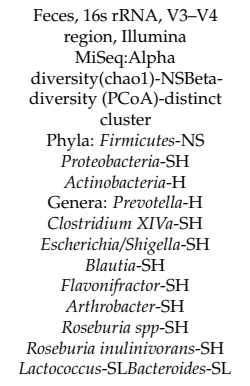 & & NS & $\begin{array}{l}\text { Epid- } \\
\text { SLInguinal- } \\
\text { NS }\end{array}$ & $\begin{array}{l}\text { ipGTT(AUC)- } \\
\text { NSITT(AUC)- } \\
\text { SL }\end{array}$ & & & $\begin{array}{l}\text { TNFa, IL-6, } \\
\text { MCP-1-SL }\end{array}$ & & \\
\hline 17 & $\begin{array}{l}\text { Wang et al., } \\
2019 \text { [52] }\end{array}$ & $\begin{array}{c}\text { ICR mice, } \mathrm{M}, \\
5-6 \mathrm{~W}, \\
(29-3 \mathrm{~g})\end{array}$ & $\begin{array}{c}\text { 6/grp } \\
\text { NDHFHFD + } \\
\text { CA }\end{array}$ & $p$ & $6 \mathrm{w}$ & $18.40 \%$ & $\begin{array}{c}\text { Chlorogenic } \\
\text { acidid(CA)- } \\
150 \\
\mathrm{mg} / \mathrm{kg} / \mathrm{day}\end{array}$ & $\begin{array}{l}\text { Oral } \\
\text { gav- } \\
\text { age }\end{array}$ & 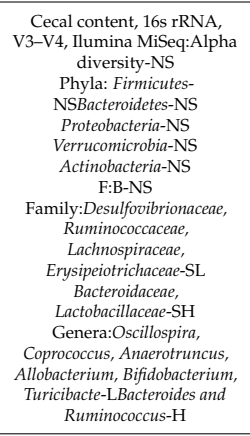 & & $\mathrm{SL}$ & Epid-SL & . & & & & $\begin{array}{c}\text { TC-SL } \\
\text { TAG-SL } \\
\text { LDL-SL } \\
\text { HDL-SH }\end{array}$ & \\
\hline 18 & $\begin{array}{l}\text { W. Liao } \\
\text { et al., 2018 } \\
\text { [53] }\end{array}$ & $\begin{array}{c}\mathrm{C} 57 \mathrm{BL} / 6 \mathrm{~J} \\
\text { mice, } \mathrm{M}, \\
8 \mathrm{w}\end{array}$ & $\begin{array}{c}\text { 7-8/grp } \\
\text { NDNDD+RHFD } \\
\text { HFD +R } \\
\text { HFD + FT } \\
*^{*} \text { only results } \\
\text { for HFD+R } \\
\text { included in this } \\
\text { review }\end{array}$ & $p$ & $4 \mathrm{w}$ & $60 \%$ & $\begin{array}{l}\text { Resveratrol } \\
\text { (R) - 400 } \\
\mathrm{mg} / \mathrm{g} \text { in } \\
\text { diet }\end{array}$ & $\begin{array}{c}\text { Within } \\
\text { diet }\end{array}$ & 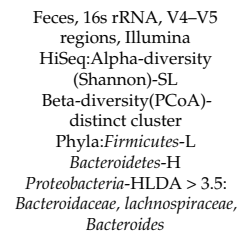 & NS & $\mathrm{SL}$ & $\begin{array}{l}\text { Perigonadal- } \\
\text { SL } \\
\text { Inguinal-SL }\end{array}$ & $\begin{array}{c}\text { OGTT(AUC): } \\
\text { SL } \\
\text { IpITT (AUC)-SL }\end{array}$ & & & & & \\
\hline
\end{tabular}


Table 2. Cont.

\begin{tabular}{|c|c|c|c|c|c|c|c|c|c|c|c|c|c|c|c|c|c|c|}
\hline No. & $\begin{array}{l}\text { Author, } \mathrm{Yr}_{\mathrm{r}} \\
\text { [Reference] }\end{array}$ & $\begin{array}{l}\text { Species, } \\
\text { Sex, Age } \\
\text { (w)/ } \\
\text { Weight (g) }\end{array}$ & $\begin{array}{l}\text { Number of } \\
\text { Animals, } n / \\
\text { Groups (Grp) }\end{array}$ & $\begin{array}{l}\text { Prevention } \\
\text { (p)/Treatment } \\
\text { (T) }\end{array}$ & $\begin{array}{l}\text { Duration of } \\
\text { Intervention } \\
\text { (d/wks/mths) }\end{array}$ & $\begin{array}{l}\text { HFD } \\
(\% \mathrm{Fat})\end{array}$ & Dosage & MOA & $\begin{array}{l}\text { Sample/Method/ } \\
\text { Hypervariable Region/GM } \\
\text { Composition }\end{array}$ & $\begin{array}{l}\text { Energy//Food } \\
\text { Intake }\end{array}$ & Weight & $\begin{array}{l}\text { VAT/ } \\
\text { SAT }\end{array}$ & $\begin{array}{c}\text { Glucose } \\
\text { (FG/GTT/ITT) }\end{array}$ & Hormones & НОМА-IR & Adipocytokine & Lipid Profile & Endotoxins \\
\hline & \multicolumn{18}{|c|}{ PURE COMPOUNDS-RATS } \\
\hline 19 & $\begin{array}{l}\text { L. Zhao } \\
\text { et al., 2017 } \\
\text { [54] }\end{array}$ & $\begin{array}{l}\text { Wistar rats, } \\
\mathrm{M}, \\
(160-180 \mathrm{~g})\end{array}$ & $\begin{array}{l}\text { 8/grp NDHFD } \\
\text { HFD + QR }\end{array}$ & $\mathrm{T}$ & $8 \mathrm{w}$ & $45 \%$ & $\begin{array}{l}\text { Combination } \\
\text { of Quercetin } \\
\text { (Q) - - 30 } \\
\mathrm{mg} / \mathrm{kg} \text { body } \\
\text { weight/day } \\
\text { Resverartal } \\
\text { (R)-15 } \\
\mathrm{mg} / \mathrm{kg} \text { body } \\
\text { weight/day }\end{array}$ & $\begin{array}{l}\text { Oral } \\
\text { gav- } \\
\text { age }\end{array}$ & 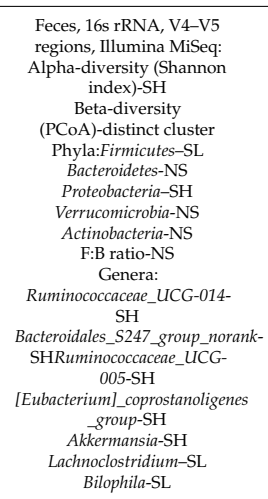 & NS & $\mathrm{SL}$ & $\begin{array}{l}\text { Perinephric- } \\
\text { SLEpid- } \\
\text { SLSAT: } \\
\text { NS }\end{array}$ & & SL & & $\begin{array}{l}\text { Leptin- } \\
\text { NSAdiponectin- } \\
\text { SHTNFa-SL } \\
\text { IL-6-SL } \\
\text { MCP-1-SL }\end{array}$ & $\begin{array}{l}\text { TC-SL } \\
\text { TAG-SL } \\
\text { HDL-SH } \\
\text { LDL-SL }\end{array}$ & \\
\hline 20 & $\begin{array}{l}\text { C. Yang } \\
\text { et al.,2019 } \\
\text { [55] }\end{array}$ & $\begin{array}{l}\text { Wistar rats, } \\
\mathrm{M}, \\
5 \mathrm{w}\end{array}$ & $\begin{array}{c}\text { 6/grp } \\
\text { NDHFDHFD + } \\
\text { RHFD + SHFD } \\
+ \text { C }\end{array}$ & $p$ & $8 \mathrm{w}$ & $45 \%$ & 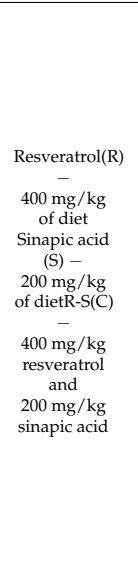 & $\begin{array}{c}\text { Within } \\
\text { diet }\end{array}$ & 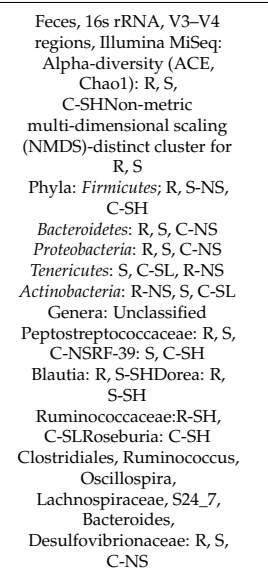 & R,S, C-NS & $\begin{array}{l}\text { R,S, } \\
\text { C-NS }\end{array}$ & & $\begin{array}{l}\text { FG: R-SL } \\
\text { OGTT(AUC): R, } \\
\text { S, C-NS }\end{array}$ & $\begin{array}{l}\text { FI-R, S, } \\
\text { C-NS }\end{array}$ & R, C-SL & & 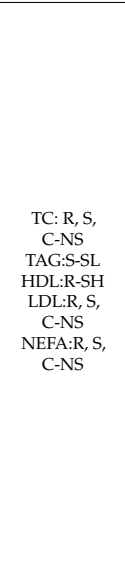 & \\
\hline
\end{tabular}


Table 2. Cont.

\begin{tabular}{|c|c|c|c|c|c|c|c|c|c|c|c|c|c|c|c|c|c|c|}
\hline No. & $\begin{array}{l}\text { Author, Yr, } \\
\text { [Reference] }\end{array}$ & $\begin{array}{c}\text { Species, } \\
\text { Sex, Age } \\
\text { (w)/ } \\
\text { Weight (g) }\end{array}$ & $\begin{array}{c}\text { Number of } \\
\text { Animals, } n \\
\text { Groups (Grp) }\end{array}$ & $\begin{array}{l}\text { Prevention } \\
(p) / / \text { Treatment } \\
\text { (T) }\end{array}$ & $\begin{array}{l}\text { Duration of } \\
\text { Intervention } \\
\text { (d/wks/mths) }\end{array}$ & $\begin{array}{l}\mathrm{HFD} \\
(\% \text { Fat })\end{array}$ & Dosage & MOA & $\begin{array}{c}\text { Sample/Method/ } \\
\text { Hypervariable Region/GM } \\
\text { Composition }\end{array}$ & $\begin{array}{l}\text { Energy/Food } \\
\text { Intake }\end{array}$ & Weight & $\begin{array}{l}\mathrm{VAT} / \\
\mathrm{SAT}\end{array}$ & $\begin{array}{c}\text { Glucose } \\
\text { (FG/GTT/ITT) }\end{array}$ & Hormones & HOMA-IR & Adipocytokine & Lipid Profile & Endotoxins \\
\hline 21 & $\begin{array}{l}\text { Luo et al., } \\
2019[56]\end{array}$ & $\begin{array}{c}\text { Sprague } \\
\text { Dawley rats, } \\
\text { M, } \\
5 \mathrm{w}\end{array}$ & $\begin{array}{l}\text { 16/grp ND } \\
\text { HFDHFD+ } \\
\text { SLIFHFD + } \\
\text { HSIF }\end{array}$ & $\mathrm{T}$ & $4 \mathrm{w}$ & $\begin{array}{c}\mathrm{ND}+ \\
15 \% \text { pork } \\
\text { fat }\end{array}$ & $\begin{array}{l}\mathrm{LSIF}=150 \\
\mathrm{mg} / \mathrm{kg} \text { soy } \\
\text { isoflavone } \\
\text { (SIF)HSII = } \\
\text { 450 } \\
\mathrm{mg} / \mathrm{kg}(\mathrm{SIF})\end{array}$ & $\begin{array}{c}\text { Oral } \\
\text { gav- } \\
\text { age }\end{array}$ & 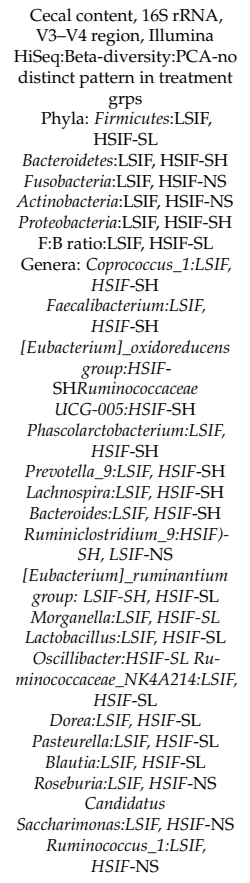 & $\begin{array}{c}\text { LSIF, } \\
\text { HSIF-NS }\end{array}$ & $\begin{array}{l}\text { LSIF, } \\
\text { HSIF-SL }\end{array}$ & & & & & & $\begin{array}{c}\text { TC:LSIF-NS, } \\
\text { HSIF-SL } \\
\text { TAG:LSIF, } \\
\text { HSSF-NS } \\
\text { HDL:LSIF- } \\
\text { NS, HSIF-SL } \\
\text { LDL:LSIF- } \\
\text { NS, } \\
\text { HSIF-SL }\end{array}$ & $\begin{array}{l}\text { LPS: LSIF, } \\
\text { HSIF-SL }\end{array}$ \\
\hline \multicolumn{19}{|c|}{ COMBINATION OF PURE COMPOUNDS } \\
\hline 22 & $\begin{array}{l}\text { Yong-Feng } \\
\text { et al.,2019 } \\
{[57]}\end{array}$ & $\begin{array}{c}\mathrm{C} 57 \mathrm{BL} / 6 \mathrm{~J} \\
\text { mice, } \mathrm{M}, 8 \mathrm{w}\end{array}$ & $\begin{array}{c}\text { 12/grp } \\
\text { NDHFHFD + } \\
\text { TFQ }\end{array}$ & $p$ & $12 \mathrm{w}$ & $60 \%$ & $\begin{array}{l}\text { Total } \\
\text { flavonoids } \\
\text { of Qunhhou } \\
\text { (FF)--300 } \\
\mathrm{mg} / \mathrm{kg} / \text { day }\end{array}$ & $\begin{array}{l}\text { Oral } \\
\text { gav- } \\
\text { age }\end{array}$ & 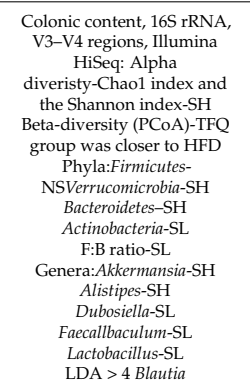 & & $\mathrm{SL}$ & Epid-SL & $\begin{array}{l}\text { FG-SL } \\
\text { OGTT(AUC)-SL } \\
\text { ITT(AUC)-NS }\end{array}$ & Fl-NS & SL & & $\begin{array}{l}\text { Serum: } \\
\text { TC-SL } \\
\text { TAG-SL } \\
\text { LLD-SL } \\
\text { HDL-NS } \\
\text { NEFA-SL } \\
\text { LiverTC-SS } \\
\text { TAG-SL }\end{array}$ & LPS-SL \\
\hline
\end{tabular}


Table 2. Cont.

\begin{tabular}{|c|c|c|c|c|c|c|c|c|c|c|c|c|c|c|c|c|c|c|}
\hline No. & $\begin{array}{l}\text { Author, } \mathrm{Y}_{\mathbf{r}} \\
\text { [Reference] }\end{array}$ & $\begin{array}{c}\text { Species, } \\
\text { Sex, Age } \\
\text { (w)/ } \\
\text { Weight (g) }\end{array}$ & $\begin{array}{l}\text { Number of } \\
\text { Animals, } n / \\
\text { Groups (Grp) }\end{array}$ & $\begin{array}{l}\text { Prevention } \\
(p) / / \text { Treatment } \\
\text { (T) }\end{array}$ & $\begin{array}{l}\text { Duration of } \\
\text { Intervention } \\
\text { (d/wks/mths) }\end{array}$ & $\begin{array}{l}\mathrm{HFD} \\
(\% \mathrm{Fat})\end{array}$ & Dosage & MOA & $\begin{array}{c}\text { Sample/Method/ } \\
\text { Hypervariable Region/GM } \\
\text { Composition }\end{array}$ & $\begin{array}{c}\text { Energy//Food } \\
\text { Intake }\end{array}$ & Weight & $\begin{array}{l}\mathrm{VAT} / \mathrm{I} \\
\mathrm{SAT}\end{array}$ & $\begin{array}{c}\text { Glucose } \\
\text { (FG/GTT/ITT) }\end{array}$ & Hormones & HOMA-IR & Adipocytokine & Lipid Profile & Endotoxins \\
\hline \multirow[t]{2}{*}{23} & $\begin{array}{l}\text { Zhu et al., } \\
2018 \text { [58] }\end{array}$ & $\begin{array}{c}\text { Sprague- } \\
\text { Dawley rats, } \\
\mathrm{M} \\
(120-140 \mathrm{~g})\end{array}$ & $\begin{array}{c}\text { 6/grp } \\
\text { NDND+LPT } \\
\text { ND + MPT ND } \\
+ \text { + HPTHCHC + } \\
\text { LPTHC + MPT } \\
\text { HC + HPT }\end{array}$ & $\mathrm{T}$ & $2 \mathrm{wks}$ & $\begin{array}{c}\text { HC } \\
\text { diet- } \\
81.8 \% \\
\text { basic } \\
\text { diet, } \\
6 \% \\
\text { dried } \\
\text { egg } \\
\text { yolk, } \\
5 \% \\
\text { full } \\
\text { cream } \\
\text { milk } \\
\text { pow- } \\
\text { der, } \\
5 \% \\
\text { lard, } \\
2 \% \\
\text { choles- } \\
\text { terol, } \\
\text { and } \\
0.2 \% \\
\text { sodium } \\
\text { cholate }\end{array}$ & $\begin{array}{c}\text { Low } \\
\text { perimmon } \\
\text { tannin } \\
\text { (LPT)-50 } \\
\text { mg/kg } \\
\text { bwMedium } \\
\text { persimmon } \\
\text { tannin } \\
\text { (MPT)-100 } \\
\text { mg kg } \\
\text { bwHigh } \\
\text { persimmon } \\
\text { tannin } \\
\text { (HPT)-200 } \\
\text { mg/kg bw }\end{array}$ & $\begin{array}{l}\text { Oral } \\
\text { gav- } \\
\text { age }\end{array}$ & 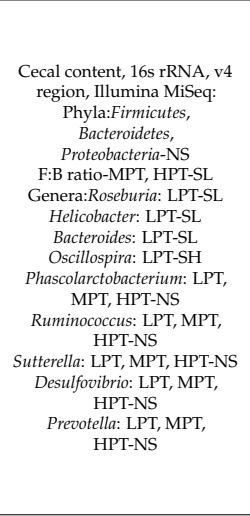 & $\begin{array}{l}\text { LPT, MPT, } \\
\text { HPT-NS }\end{array}$ & $\begin{array}{l}\text { LPT, } \\
\text { MPT, } \\
\text { HPT-NS }\end{array}$ & & & & 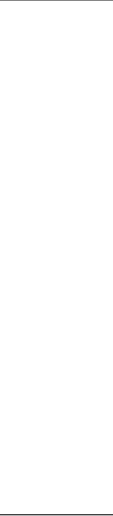 & & $\begin{array}{l}\text { Serum:TC: } \\
\text { LPT, HPT-SL } \\
\text { TAG:MPT- } \\
\text { SL } \\
\text { LDL: LPT-SL } \\
\text { Liver:TC: } \\
\text { LPT, HPT, } \\
\text { MPT-NS } \\
\text { TAG:LP, } \\
\text { HPT, } \\
\text { MPT-NS }\end{array}$ & \\
\hline & \multicolumn{18}{|c|}{ PHENOLIC EXTRACTS-MICE } \\
\hline 24 & $\begin{array}{l}\text { H. Lee et al., } \\
2019 \text { [59] }\end{array}$ & $\begin{array}{l}\text { C57BL/6Nmice, } \\
\mathrm{M}, 5 \mathrm{w}\end{array}$ & $\begin{array}{c}n=\text { NAND- } \\
\text { HFDHFD }+ \\
\text { RO125HFD + } \\
\text { RO250 }\end{array}$ & $\mathrm{T}$ & $16 \mathrm{w}$ & $45 \%$ & $\begin{array}{c}\text { Rubus occi- } \\
\text { dentalis(RO) } \\
\text { 125: 125 } \\
\mathrm{mg} / \mathrm{kg} / \text { day; } \\
\text { RO250: } 250 \\
\mathrm{mg} / \mathrm{kg} / \text { day }\end{array}$ & $\begin{array}{l}\text { Oral } \\
\text { gav- } \\
\text { age }\end{array}$ & 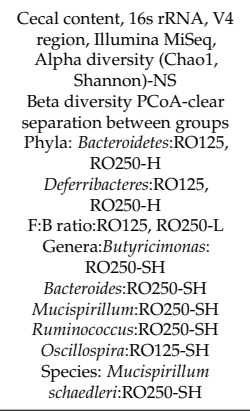 & & NS & & $\begin{array}{c}\text { iPGTT } \\
(\mathrm{AUC}) \mathrm{RO} 250- \\
\mathrm{SL}\end{array}$ & & & & & \\
\hline 25 & $\begin{array}{l}\text { Collins et al. } \\
2016[60]\end{array}$ & $\begin{array}{c}\mathrm{C} 57 \mathrm{BL} / 6 \mathrm{~J} \\
\text { mice, } \mathrm{M}, 4 \mathrm{w}\end{array}$ & $\begin{array}{c}\text { 10/grp ND } \\
\text { HFDHFD + } \\
\text { EPHFD + } \\
\text { NEPHFD + } \\
\text { EP-NEPHFD + } \\
\text { GP }\end{array}$ & $p$ & $16 \mathrm{w}$ & $44 \%$ & 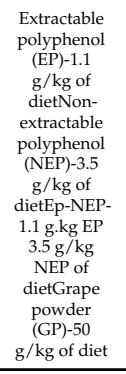 & $\begin{array}{c}\text { Within } \\
\text { diet }\end{array}$ & 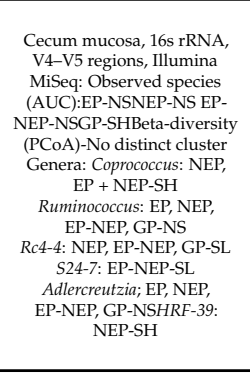 & & $\begin{array}{c}\text { EP- } \\
\text { SLNEP- } \\
\text { SL } \\
\text { EP-NEP- } \\
\text { SL } \\
\text { GP-NS }\end{array}$ & $\begin{array}{l}\text { \% body fat } \\
\text { (wk 15): } \\
\text { EP-SL } \\
\text { NNPF-NS } \\
\text { EP-NEP-SL } \\
\text { GPPS } \\
\text { WATEP-SL } \\
\text { NAPP-SL } \\
\text { EP-NPE-SL } \\
\text { GP-NS }\end{array}$ & $\begin{array}{c}\text { IpGTT } \\
\text { (AUC):EP-SL } \\
\text { NEP-SL EP- } \\
\text { NEP-SLGP-NS }\end{array}$ & & $\begin{array}{l}\text { EP-SLNEP- } \\
\text { NS } \\
\text { EP-NEP- } \\
\text { SLGP-NS }\end{array}$ & $\begin{array}{l}\text { Plasma MCP-1: } \\
\text { NEP-NS EP- } \\
\text { NEP-SLGP-NS }\end{array}$ & 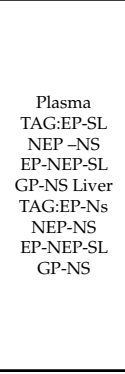 & $\begin{array}{c}\text { Plasma } \\
\text { LBP:EP-NS } \\
\text { NEP-SL } \\
\text { EP-NEP-SL } \\
\text { GP-NS }\end{array}$ \\
\hline
\end{tabular}


Table 2. Cont.

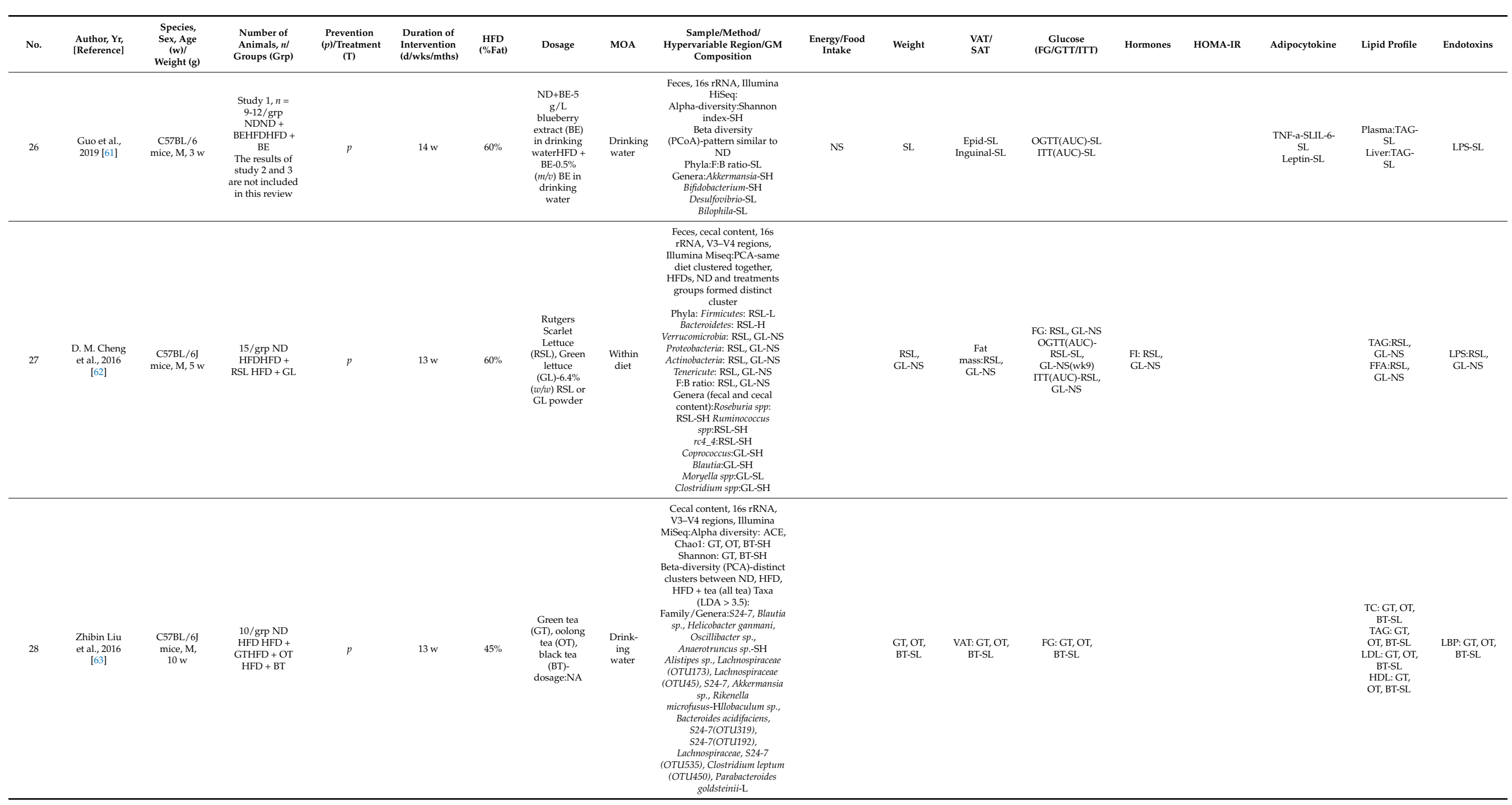


Table 2. Cont.

\begin{tabular}{|c|c|c|c|c|c|c|c|c|c|c|c|c|c|c|c|c|c|c|}
\hline No. & $\begin{array}{l}\text { Author, , r, } \\
\text { [Referencel }\end{array}$ & $\begin{array}{c}\text { Species, } \\
\text { Sex, Age } \\
\text { (w)l } \\
\text { Weight (g) } \\
\end{array}$ & $\begin{array}{c}\text { Number of } \\
\text { Animals }, n l \\
\text { Groups (Grp) }\end{array}$ & $\begin{array}{l}\text { Prevention } \\
(p) / / \text { Treatment } \\
\text { (T) }\end{array}$ & $\begin{array}{l}\text { Duration of } \\
\text { Intervention } \\
\text { (d/wks/mths) }\end{array}$ & $\begin{array}{c}\text { HFD } \\
\text { (\%Fat) }\end{array}$ & Dosage & MOA & $\begin{array}{c}\text { Sample/Method/ } \\
\text { Hypervariable Region/GM } \\
\text { Composition }\end{array}$ & $\begin{array}{l}\text { Energy//Food } \\
\text { Intake }\end{array}$ & Weight & $\begin{array}{l}\text { VAT/ } \\
\text { SAT }\end{array}$ & $\begin{array}{l}\text { Glucose } \\
\text { (FG/GTT/ITT) }\end{array}$ & Hormones & HOMA-IR & Adipocytokine & Lipid Profile & Endotoxins \\
\hline 29 & $\begin{array}{l}\text { Griffin et al., } \\
2017 \text { [64] }\end{array}$ & $\begin{array}{c}\mathrm{C} 57 \mathrm{BL} / 6 \mathrm{~J} \\
\text { mice, } \mathrm{M}, 9 \mathrm{w}\end{array}$ & $\begin{array}{l}\text { 8/grp } \\
\text { NDHFDHFD } \\
\text { 10HFD100 }\end{array}$ & $\mathrm{T}$ & $12 \mathrm{w}$ & $45 \%$ & $\begin{array}{c}\text { HFD10 100 } \\
\text { grape sed } \\
\text { extract (GE) } \\
\text { mg/kg/day } \\
\text { HFD100 } \\
100 \\
\text { mg } / \mathrm{kg} / \text { day }\end{array}$ & $\begin{array}{c}\text { Within } \\
\text { diet }\end{array}$ & 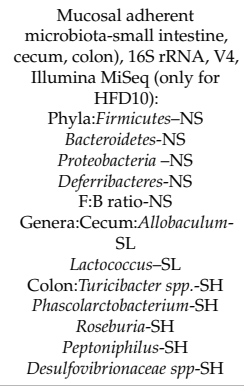 & $\begin{array}{l}\text { HFD10-NS } \\
\text { HFD100-NS }\end{array}$ & $\begin{array}{l}\text { HFD10- } \\
\text { NS } \\
\text { NFD100- } \\
\text { NS }\end{array}$ & $\begin{array}{c}\text { Total body } \\
\text { fat:HFD10- } \\
\text { NS } \\
\text { HFD100-NS }\end{array}$ & $\begin{array}{c}\text { FG:HFD10-SL } \\
\text { HFD100-NS } \\
\text { OGTT(AUC): } \\
\text { HFD10-SL } \\
\text { HFD100-NS } \\
\text { ITT } \\
\text { (AUC):HFD10- } \\
\text { NS } \\
\text { HFD100-NS }\end{array}$ & & & & & \\
\hline 30 & $\begin{array}{l}\text { Y. Li et al., } \\
2019 \text { [65] }\end{array}$ & $\begin{array}{c}\text { C57BL/6J } \\
(\mathrm{B} 6 \text { mice, } \mathrm{M}, \\
(22 \pm 2 \mathrm{~g})\end{array}$ & $\begin{array}{c}\text { 10/grp } \\
\text { NDHFDHFD + } \\
\text { CROHFD + } \\
\text { SRO }\end{array}$ & $p$ & $12 \mathrm{w}$ & $24.50 \%$ & 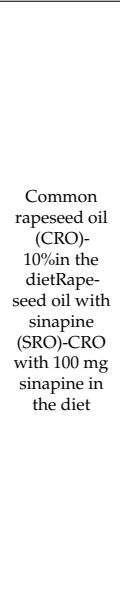 & $\begin{array}{c}\text { Within } \\
\text { diet }\end{array}$ & 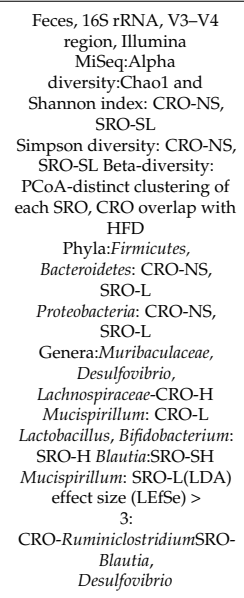 & NS & $\begin{array}{l}\text { CRO-NS } \\
\text { SRO-SL }\end{array}$ & $\begin{array}{c}\text { Epid: } \\
\text { CRO-NS } \\
\text { SRO-SL } \\
\text { Perirenal:CRO- } \\
\text { NS } \\
\text { SRO-SL }\end{array}$ & $\begin{array}{l}\text { FG:CRO-NS } \\
\text { SRO-SL }\end{array}$ & $\begin{array}{l}\text { FI:CRO- } \\
\text { NS } \\
\text { SRO-SL }\end{array}$ & $\begin{array}{l}\text { CRO-NS } \\
\text { SRO-SL }\end{array}$ & & $\begin{array}{l}\text { Serum: } \\
\text { TAGGCRR- } \\
\text { NS,SRO-SL } \\
\text { LDLCCRO, } \\
\text { SLO-SL } \\
\text { Liver: VLLL: } \\
\text { CRO-NS, } \\
\text { SRO-SL }\end{array}$ & \\
\hline
\end{tabular}


Table 2. Cont.

\begin{tabular}{|c|c|c|c|c|c|c|c|c|c|c|c|c|c|c|c|c|c|c|}
\hline No. & $\begin{array}{l}\text { Author, } \mathrm{Yr}_{\mathbf{r}} \\
\text { [Referencel }\end{array}$ & $\begin{array}{c}\text { Species, } \\
\text { Sex, Age } \\
\text { (w)l } \\
\text { Weight (g) }\end{array}$ & $\begin{array}{l}\text { Number of } \\
\text { Animals, } n / \\
\text { Groups (Grp) }\end{array}$ & $\begin{array}{l}\text { Prevention } \\
(p) / / \text { Treatment } \\
\text { (T) }\end{array}$ & $\begin{array}{l}\text { Duration of } \\
\text { Intervention } \\
\text { (d/ws/mths) }\end{array}$ & $\begin{array}{l}\mathrm{HFD} \\
(\% \mathrm{Fat})\end{array}$ & Dosage & MOA & $\begin{array}{c}\text { Sample/Method/ } \\
\text { Hypervariable Region/GM } \\
\text { Composition }\end{array}$ & $\begin{array}{c}\text { Energy/Food } \\
\text { Intake }\end{array}$ & Weight & $\begin{array}{l}\text { VAT/ } \\
\text { SAT }\end{array}$ & $\begin{array}{c}\text { Glucose } \\
\text { (FG/GTT/ITT) }\end{array}$ & Hormones & HOMA-IR & Adipocytokine & Lipid Profile & Endotoxins \\
\hline 31 & $\begin{array}{l}\text { Ma et al., } \\
2019 \text { [66] }\end{array}$ & $\begin{array}{c}\mathrm{C} 57 \mathrm{BL} / 6 \\
\text { mice, } \mathrm{M}, 6 \mathrm{w}\end{array}$ & $\begin{array}{l}\text { 8/grp ND } \\
\text { HFHFD + } \\
\text { TPHHFD }+ \\
\text { TPMHFD+ } \\
\text { TPH }\end{array}$ & $p$ & $12 \mathrm{w}$ & $36.71 \%$ & $\begin{array}{c}\text { Tea } \\
\text { polyphenol } \\
\text { lowdose } \\
\text { (TIL)-100 } \\
\text { mg/kg/day } \\
\text { Tea } \\
\text { polyphenol } \\
\text { medium } \\
\text { dose } \\
\text { (TPM)-200 } \\
\mathrm{mg} / \mathrm{kg} \text { /day } \\
\text { Tea } \\
\text { polyphenol } \\
\text { high dose } \\
\text { (TPH)-400 } \\
\mathrm{mg} / \mathrm{kg} / \text { day }\end{array}$ & $\begin{array}{l}\text { Oral } \\
\text { gave } \\
\text { age }\end{array}$ & 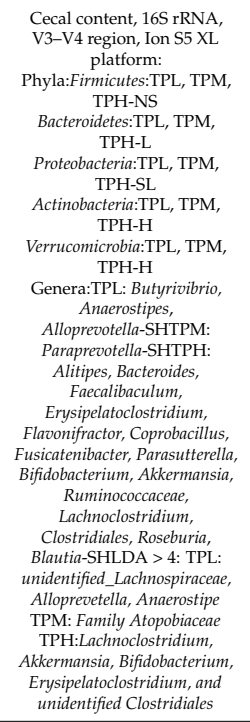 & & $\begin{array}{c}\text { TPL, } \\
\text { TPM, } \\
\text { TPH-SL }\end{array}$ & & $\begin{array}{l}\text { FG:TPL, TPM, } \\
\text { TPH-NS }\end{array}$ & $\begin{array}{l}\text { FI:TPL, } \\
\text { TPM-SL, } \\
\text { TPH-NS }\end{array}$ & $\begin{array}{l}\text { TPL.TPM- } \\
\text { SL, } \\
\text { TPH-NS }\end{array}$ & $\begin{array}{l}\text { TNF- } \alpha \text { : TPL, } \\
\text { TPM, } \\
\text { TPH-SLIL-6: } \\
\text { TPL, TPM, } \\
\text { TPH-SL }\end{array}$ & $\begin{array}{l}\text { TC:TPL, } \\
\text { TPM,, } \\
\text { TPH-NS } \\
\text { TAG:TPL, } \\
\text { TPM, } \\
\text { TPH-NS } \\
\text { HDL:TPL, } \\
\text { TPM, } \\
\text { TPH-SH } \\
\text { LDL:TPL- } \\
\text { NS, TPM, } \\
\text { TPH-SL }\end{array}$ & $\begin{array}{l}\text { LPSS:TPL, } \\
\text { TPM-NS, } \\
\text { TPH-SL }\end{array}$ \\
\hline 32 & $\begin{array}{l}\text { Van Hul } \\
\text { et al., 2018 } \\
{[67]}\end{array}$ & $\begin{array}{c}\mathrm{C} 57 \mathrm{BL} / 6 \mathrm{~J} \\
\text { mice, } \mathrm{M}, 9 \mathrm{w}\end{array}$ & $\begin{array}{c}\text { 14/grp } \\
\text { NDHFDHFD + } \\
\text { CBE GPD + } \\
\text { GPE }\end{array}$ & $p$ & $8 \mathrm{w}$ & $60 \%$ & $\begin{array}{c}\text { Cinnamon } \\
\text { bark extract } \\
\text { (CBE)-2 } \\
\text { g/kg grape } \\
\text { pomace } \\
\text { extract } \\
\text { (GPF)-8.2 } \\
\mathrm{g} / \mathrm{kg}\end{array}$ & $\begin{array}{c}\text { Within } \\
\text { diet }\end{array}$ & 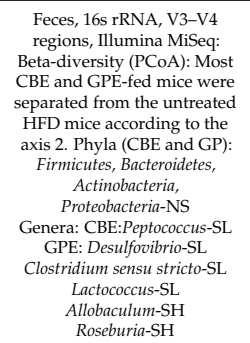 & $\begin{array}{l}\text { CBE, } \\
\text { GPE-NS }\end{array}$ & $\begin{array}{c}\text { CBE, } \\
\text { GPE-NS }\end{array}$ & $\begin{array}{l}\text { VAT: CBE, } \\
\text { GPE-NS } \\
\text { SAT CBE, } \\
\text { GPE-NS }\end{array}$ & $\begin{array}{l}\text { OGTT (AUC): } \\
\text { CBE-SL } \\
\text { GPE-NS }\end{array}$ & $\begin{array}{l}\text { FI:CBE, } \\
\text { GPE-NS }\end{array}$ & $\begin{array}{l}\text { IR-index: } \\
\text { CBE-NS } \\
\text { GPE-SL }\end{array}$ & 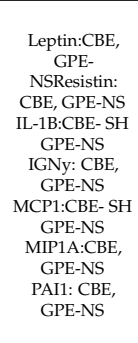 & $\begin{array}{c}\text { Plasma:TC, } \\
\text { TAG, } \\
\text { NEFA:CBE, } \\
\text { GPE-NS } \\
\text { Liver:TC:CBE, } \\
\text { GPE-NS } \\
\text { TAG-CBE- } \\
\text { NS, } \\
\text { GPE-SL }\end{array}$ & \\
\hline
\end{tabular}


Table 2. Cont.

\begin{tabular}{|c|c|c|c|c|c|c|c|c|c|c|c|c|c|c|c|c|c|c|}
\hline No. & $\begin{array}{l}\text { Author, } \mathrm{Yr}_{\mathrm{r}} \\
\text { [Reference] }\end{array}$ & $\begin{array}{c}\text { Species, } \\
\text { Sex, Age } \\
\text { (w)/ } \\
\text { Weight (g) }\end{array}$ & $\begin{array}{l}\text { Number of } \\
\text { Animals, } n / \\
\text { Groups (Grp) }\end{array}$ & $\begin{array}{l}\text { Prevention } \\
(p) / \text { Treatment } \\
\text { (T) }\end{array}$ & $\begin{array}{l}\text { Duration of } \\
\text { Intervention } \\
\text { (d/wks/mths) }\end{array}$ & $\begin{array}{l}\mathrm{HFD} \\
(\% \text { Fat) }\end{array}$ & Dosage & MOA & $\begin{array}{c}\text { Sample/Method/ } \\
\text { Hypervariable Region/GM } \\
\text { Composition }\end{array}$ & $\begin{array}{l}\text { Energy//Food } \\
\text { Intake }\end{array}$ & Weight & $\begin{array}{l}\mathrm{VAT} / \\
\mathrm{SAT}\end{array}$ & $\begin{array}{c}\text { Glucose } \\
\text { (FG/GTT/ITT) }\end{array}$ & Hormones & HOMA-IR & Adipocytokine & Lipid Profile & Endotoxins \\
\hline 33 & $\begin{array}{l}\text { Anhe et al., } \\
2015[68]\end{array}$ & 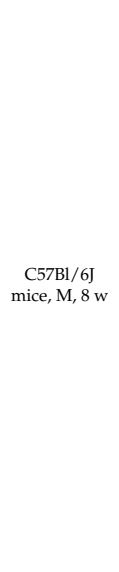 & $\begin{array}{l}12 / \text { grp } \\
\text { NDHFHS } \\
\mathrm{HFHS}+\mathrm{CE}\end{array}$ & $p$ & $8 w$ & $65 \%$ & $\begin{array}{l}\text { Cranberry } \\
\text { powdered } \\
\text { extract } \\
\text { (CE)-200 } \\
\mathrm{mg} / \mathrm{kg}\end{array}$ & $\begin{array}{l}\text { Oral } \\
\text { gav- } \\
\text { age }\end{array}$ & 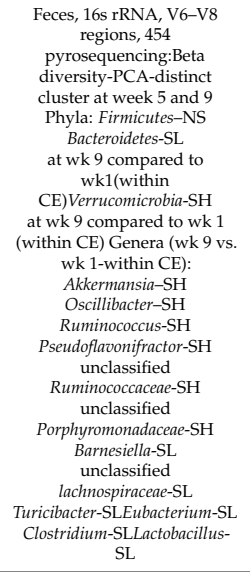 & SL & $\mathrm{SL}$ & $\begin{array}{l}\text { VAT: SL } \\
\text { SAT: NS }\end{array}$ & $\begin{array}{c}\text { FG-NS } \\
\text { OGTT(AUC)- } \\
\text { NS } \\
\text { ITT(AUC):SL }\end{array}$ & $\begin{array}{c}\text { FI-SL } \\
\text { C- } \\
\text { peptide } \\
\text { AUC-SL }\end{array}$ & SL & & $\begin{array}{l}\text { Plasma:TC- } \\
\text { SL } \\
\text { TAG-SL } \\
\text { Liver:TAG- } \\
\text { SL } \\
\text { Jejunum:TAG- } \\
\text { SL }\end{array}$ & SL \\
\hline 34 & $\begin{array}{l}\text { Anhêe tal., } \\
2017[69]\end{array}$ & $\begin{array}{c}\mathrm{C578 \textrm {Bl } / 6 \mathrm { J }} \\
\text { mice, } \mathrm{M}, 8 \mathrm{w}\end{array}$ & $\begin{array}{l}\text { 8-11/grp ND } \\
\text { ND + } \\
\text { CEHFHSHFHS } \\
+ \text { CE }\end{array}$ & $\mathrm{T}$ & $8 \mathrm{w}$ & $65 \%$ & $\begin{array}{c}\text { Cranberry } \\
\text { extract } \\
(\mathrm{C})-200 \\
\mathrm{mg} / \mathrm{kg}\end{array}$ & $\begin{array}{l}\text { Oral } \\
\text { gav- } \\
\text { age }\end{array}$ & 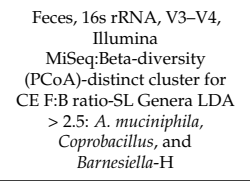 & NS & NS & $\begin{array}{l}\text { VAT: NS } \\
\text { SATT: NS }\end{array}$ & $\begin{array}{c}\text { OGTT (AUC): } \\
\text { NS } \\
\text { ipITT (AUC):SL }\end{array}$ & & & & $\begin{array}{c}\text { Plasma: } \\
\text { TAG-NS } \\
\text { LiverTAG- } \\
\text { SL }\end{array}$ & \\
\hline 35 & $\begin{array}{l}\text { Anhe et al., } \\
2018[70]\end{array}$ & 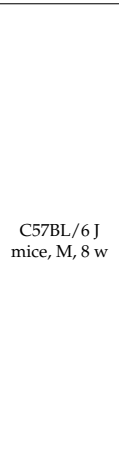 & $\begin{array}{c}\text { 12/grp } \\
\text { NDHFHSHFHS } \\
\text { + BBEHFHS + } \\
\text { CLEHFHS } \\
\text { CREHFHS }+ \\
\text { ABEHFHS } \\
\text { LGE }\end{array}$ & $p$ & $8 \mathrm{w}$ & $65 \%$ & $\begin{array}{c}\text { Bog } \\
\text { blueberries } \\
\text { (BBE), } \\
\text { cloudberries } \\
\text { (CLE), } \\
\text { crowberries } \\
\text { (CRE), } \\
\text { alpine } \\
\text { bearberries } \\
\text { (ABE) and } \\
\text { lingonber- } \\
\text { ries } \\
\text { (LGE)- } \\
\text { 200 mg } \\
\text { powdered } \\
\text { extract/kg } \\
\text { body weight }\end{array}$ & $\begin{array}{l}\text { Oral } \\
\text { gav- } \\
\text { age }\end{array}$ & 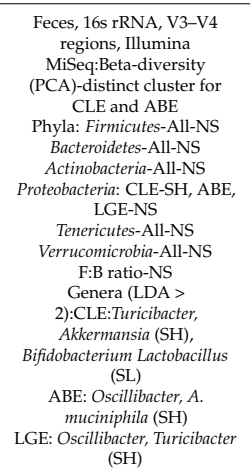 & All grps-NS & $\begin{array}{c}\text { All } \\
\text { grps-NS }\end{array}$ & $\begin{array}{l}\text { VAT: All } \\
\text { grps-NS } \\
\text { SAT: All } \\
\text { grps-NS }\end{array}$ & $\begin{array}{l}\text { FG-NS (all) } \\
\text { OGTT } \\
\text { (AUC)-NS } \\
\text { (all) } \\
\text { ITT(AUC): } \\
\text { CLE-SLC }\end{array}$ & $\begin{array}{l}\text { Fl:CLE, } \\
\text { ABE, } \\
\text { LGE- } \\
\text { SLLeptide: } \\
\text { All } \\
\text { NSAll } \\
\text { (AUC)- } \\
\text { NS }\end{array}$ & & & $\begin{array}{l}\text { Plasma:TAG: } \\
\text { CLE, ABE, } \\
\text { LGE-SL } \\
\text { Liver: TAG: } \\
\text { CLE, ABE, } \\
\text { LGE-SL }\end{array}$ & $\begin{array}{l}\text { LPS:CLE, } \\
\text { ABE, } \\
\text { LGE-SL }\end{array}$ \\
\hline
\end{tabular}


Table 2. Cont.

\begin{tabular}{|c|c|c|c|c|c|c|c|c|c|c|c|c|c|c|c|c|c|c|}
\hline No. & $\begin{array}{l}\text { Author, } \mathrm{Yr}_{\mathrm{r}} \\
\text { [Reference] }\end{array}$ & $\begin{array}{c}\text { Species, } \\
\text { Sex, Age } \\
\text { (w)/ } \\
\text { Weight (g) }\end{array}$ & $\begin{array}{l}\text { Number of } \\
\text { Animals, } n / \\
\text { Groups (Grp) }\end{array}$ & $\begin{array}{l}\text { Prevention } \\
\text { (p)/Treatment } \\
\text { (T) }\end{array}$ & $\begin{array}{l}\text { Duration of } \\
\text { Intervention } \\
\text { (d/wks/mths) }\end{array}$ & $\underset{(\% F \mathrm{Fat})}{\mathrm{HFD}}$ & Dosage & MOA & $\begin{array}{l}\text { Sample/Method/ } \\
\text { Hypervariable Region/GM } \\
\text { Composition }\end{array}$ & $\begin{array}{l}\text { Energy//Food } \\
\text { Intake }\end{array}$ & Weight & $\begin{array}{l}\text { VAT/ } \\
\text { SAT }\end{array}$ & $\begin{array}{c}\text { Glucose } \\
\text { (FG/GTT/ITT) }\end{array}$ & Hormones & HOMA-IR & Adipocytokine & Lipid Profile & Endotoxins \\
\hline 36 & $\begin{array}{l}\text { Chen et al., } \\
2018[71]\end{array}$ & $\begin{array}{c}\text { C578L/6 } / 6 \\
\text { mice, } \mathrm{M}, 6 \mathrm{w}\end{array}$ & $\begin{array}{l}\text { 8/grp ND } \\
\text { HFDHFD+ } \\
\text { KDCHFD+ } \\
\text { FBT }\end{array}$ & $p$ & $8 w$ & $45 \%$ & $\begin{array}{l}\text { Kudingcha } \\
\text { (KDC) and } \\
\text { Furhuan } \\
\text { Brick Tea } \\
\text { (FBT)-400 } \\
\mathrm{mg} / \mathrm{kg} / \mathrm{d}\end{array}$ & Intragastric & 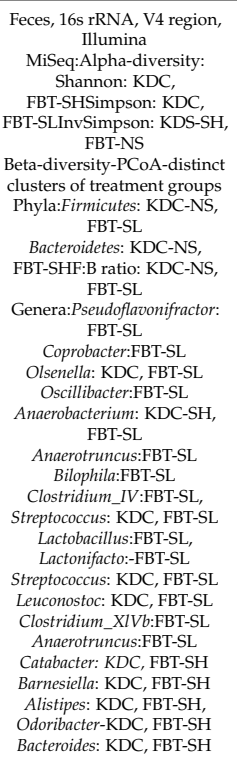 & NS & $\begin{array}{c}\text { KDC, } \\
\text { FBT-SL }\end{array}$ & $\begin{array}{l}\text { Epid: KDC, } \\
\text { BFT-SL } \\
\text { Perirenal: } \\
\text { KDC, } \\
\text { FBT-SL }\end{array}$ & & & & 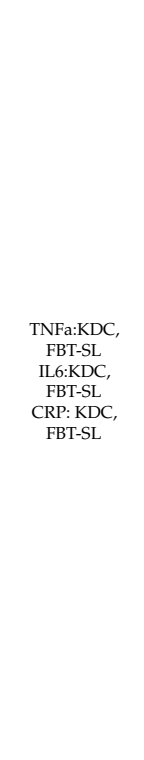 & $\begin{array}{l}\text { Serum:TC: } \\
\text { KDC-NS } \\
\text { FBT-SL } \\
\text { TAG KDC, } \\
\text { FBT-NS } \\
\text { LDL: } \\
\text { KDC-NS, } \\
\text { FBT-SL } \\
\text { HDL KDC, } \\
\text { FBT-NS, } \\
\text { Liver:TAG: } \\
\text { KDC-NS, } \\
\text { FBT-SL }\end{array}$ & $\begin{array}{l}\text { LPS:KDC, } \\
\text { FBT-SL }\end{array}$ \\
\hline 37 & $\begin{array}{l}\text { J. Xu et al., } \\
2019 \text { [72] }\end{array}$ & $\begin{array}{c}\text { C57BL/6 } \\
\text { mice, } \mathrm{M}, 7 \mathrm{w}\end{array}$ & $\begin{array}{l}\text { 12/grp ND } \\
\text { HFDHFD + J }\end{array}$ & $p$ & $8 \mathrm{w}$ & $60 \%$ & $\begin{array}{l}\text { Jamun } \\
\text { extract } \\
\text { JI-100 } \\
\mathrm{mg} / \mathrm{kg}\end{array}$ & $\begin{array}{l}\text { Oral } \\
\text { gav- } \\
\text { age }\end{array}$ & 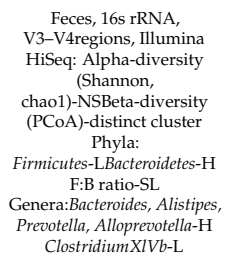 & NS & SL & $\begin{array}{l}\text { VAT:SL } \\
\text { SAT:SL }\end{array}$ & $\begin{array}{c}\text { FG-SL } \\
\text { OGTT (AUC): } \\
\text { SL } \\
\text { ITT (AUC)-SL }\end{array}$ & FI-SL & SL & & $\begin{array}{c}\text { Plasma-TC- } \\
\text { NS } \\
\text { TAG-SL } \\
\text { FAA-SH } \\
\text { Liver TC-SL } \\
\text { TAGSSL } \\
\text { FFA-SL }\end{array}$ & \\
\hline
\end{tabular}


Table 2. Cont.

\begin{tabular}{|c|c|c|c|c|c|c|c|c|c|c|c|c|c|c|c|c|c|c|}
\hline No. & $\begin{array}{l}\text { Author, } \mathrm{r}_{\mathbf{r}} \\
\text { [Referencel }\end{array}$ & $\begin{array}{c}\text { Species, } \\
\text { Sex, Age } \\
\text { (w)/ } \\
\text { Weight (g) }\end{array}$ & $\begin{array}{l}\text { Number of } \\
\text { Animals, } n / \\
\text { Groups (Grp) }\end{array}$ & $\begin{array}{l}\text { Prevention } \\
(p) / \text { Treatment } \\
\text { (T) }\end{array}$ & $\begin{array}{l}\text { Duration of } \\
\text { Intervention } \\
\text { (d/ws/mths) }\end{array}$ & $\begin{array}{c}\text { HFD } \\
(\% \text { Fat })\end{array}$ & Dosage & MOA & $\begin{array}{c}\text { Sample/Method/ } \\
\text { Hypervariable Region/GM } \\
\text { Composition }\end{array}$ & $\begin{array}{c}\text { Energy/Food } \\
\text { Intake }\end{array}$ & Weight & $\begin{array}{l}\text { VAT/ } \\
\text { SAT }\end{array}$ & $\begin{array}{c}\text { Glucose } \\
\text { (FG/GTT/ITT) }\end{array}$ & Hormones & HOMA-IR & Adipocytokine & Lipid Profile & Endotoxins \\
\hline 38 & $\begin{array}{l}\text { Dey et al., } \\
2019[73]\end{array}$ & $\begin{array}{c}\text { C57BL/6J } \\
\text { mice, } \mathrm{M}, 5 \mathrm{w}\end{array}$ & $\begin{array}{l}\text { 10/grp ND ND } \\
+ \text { GTEHFDHFD } \\
+ \text { GTE }\end{array}$ & $p$ & $8 \mathrm{w}$ & $60 \%$ & $\begin{array}{c}\text { Green tea } \\
\text { extract } \\
(\mathrm{GTE})-2 \% \\
(w / w)\end{array}$ & $\begin{array}{c}\text { Within } \\
\text { diet }\end{array}$ & 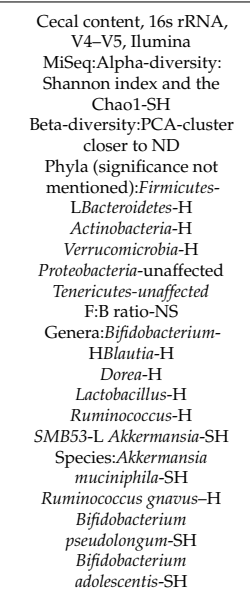 & $\mathrm{SH}$ & SL & $\begin{array}{l}\text { Epid-SL } \\
\text { RP-SL SAT: } \\
\text { SL }\end{array}$ & FG-NS & Fl-NS & $\mathrm{SL}$ & & $\begin{array}{c}\text { TC-SL } \\
\text { TG-SL } \\
\text { NEFA-SL }\end{array}$ & $\begin{array}{l}\text { LPS-NS } \\
\text { FITC- } \\
\text { dextran-SL }\end{array}$ \\
\hline 39 & $\begin{array}{l}\text { S. Wu et al., } \\
\text { 2018 [74], M. } \\
\text { Liu et al., } \\
2018 \text { [75] }\end{array}$ & $\begin{array}{l}\mathrm{C} 57 \mathrm{BL} / 6 \mathrm{~N} \\
\text { mice, } \mathrm{M}, 5 \mathrm{w}\end{array}$ & $\begin{array}{c}4 / \text { grp NDND + } \\
1 \% \\
\text { LCBPHFDHFD } \\
+0.5 \% \\
\text { LCBPFD + }+1 \% \\
\text { LCBP }\end{array}$ & $p$ & $45 \mathrm{~d}$ & $40 \%$ & $\begin{array}{l}\text { Lonicera } \\
\text { caerulea L. } \\
\text { Berry } \\
\text { Polyphenols } \\
\text { (LCBP)-0.5\% } \\
\text { and 1\% }\end{array}$ & $\begin{array}{c}\text { Within } \\
\text { diet }\end{array}$ & 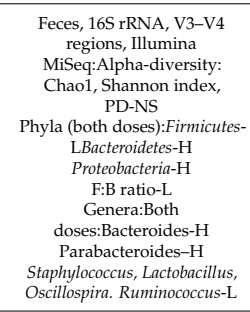 & & $\begin{array}{l}0.5 \%, 1 \% \\
\text { LCBP-SL }\end{array}$ & 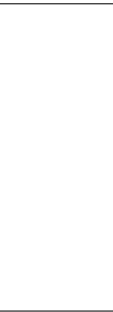 & $\begin{array}{l}\text { FG: } 0.5 \%, 1 \% \\
\text { LCBP-SL }\end{array}$ & $\begin{array}{c}\text { FI } 0.5 \% \\
1 \% \\
\text { LCBP-SL }\end{array}$ & $\begin{array}{l}0.5 \%, 1 \% \\
\text { LCBP-SL }\end{array}$ & $\begin{array}{c}\text { IL-2: } 0.5 \%, 1 \% \\
\text { LCBP-SL } \\
\text { IL-6: 0.5\%, } 1 \% \\
\text { LCBPP-SL } \\
\text { MCP1: 0.5\% } \\
\text { LCBP-NS } \\
\text { 1\% LCBP-SL } \\
\text { TNFa: 0.5\%, 1\% } \\
\text { LCBP-SL }\end{array}$ & $\begin{array}{c}\text { TAGSerum: } \\
0.5 \% \\
\text { LCBP-SL } \\
\text { 1\% LCBP-SL } \\
\text { Liver 0.5\% } \\
\text { LCBP-SL } \\
1 \% \text { LCBP-SL }\end{array}$ & $\begin{array}{l}\text { Endotoxin: } \\
\text { Serum: SL } \\
\text { (0.5.\%, } 1 \% \\
\text { LCBP) Liver. } \\
\text { SL (0.5\%, } 1 \% \\
\text { LCBP) }\end{array}$ \\
\hline 40 & $\begin{array}{l}\text { C. Wu et al. } \\
2019 \text { [76] }\end{array}$ & $\begin{array}{l}\text { C57BL/6J } \\
\text { mice, } \mathrm{M}, 8 \mathrm{w}\end{array}$ & $\begin{array}{c}8 / \\
\text { grpNDHFDHFD } \\
+ \text { PTF HFD + } \\
\text { AbHFD + Ab + } \\
\text { PTF* only } \\
\text { results for PTF } \\
\text { reported in this } \\
\text { review }\end{array}$ & $\mathrm{T}$ & $6 \mathrm{w}$ & $60 \%$ & $\begin{array}{l}\text { Pandanus } \\
\text { tectorius } \\
\text { fruitextract } \\
\text { (PTFF-200 } \\
\mathrm{mg} / \mathrm{kg} \mathrm{bw}\end{array}$ & $\begin{array}{l}\text { Oral } \\
\text { gav- } \\
\text { age }\end{array}$ & 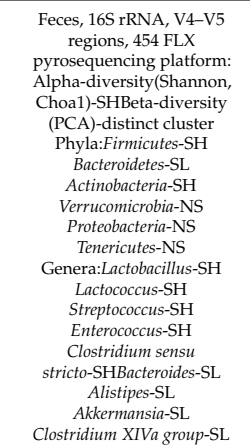 & NS & $\mathrm{SL}$ & $\begin{array}{l}\text { Epid-NS } \\
\text { SAT: NS }\end{array}$ & $\begin{array}{l}\text { FG-SL } \\
\text { OGTT (AUC): } \\
\text { SL }\end{array}$ & & & & $\begin{array}{l}\text { TC-SL } \\
\text { TG-SL } \\
\text { LDL-SL }\end{array}$ & \\
\hline
\end{tabular}


Table 2. Cont.

\begin{tabular}{|c|c|c|c|c|c|c|c|c|c|c|c|c|c|c|c|c|c|c|}
\hline No. & $\begin{array}{l}\text { Author, } \mathbf{Y r}_{\mathrm{r}} \text {, } \\
\text { [Reference] }\end{array}$ & $\begin{array}{c}\text { Species, } \\
\text { Sex, Age } \\
\text { (w)l } \\
\text { Weight (g) }\end{array}$ & $\begin{array}{c}\text { Number of } \\
\text { Animals, } n / \\
\text { Groups (Grp) }\end{array}$ & $\begin{array}{l}\text { Prevention } \\
(p) / \text { Treatment } \\
\text { (T) }\end{array}$ & $\begin{array}{l}\text { Duration of } \\
\text { Intervention } \\
\text { (d/ws/mthth) }\end{array}$ & $\begin{array}{l}\mathrm{HFD} \\
(\% \mathrm{Fat})\end{array}$ & Dosage & MOA & $\begin{array}{l}\text { Sample/Method/ } \\
\text { Hypervariable Region/GM } \\
\text { Composition }\end{array}$ & $\begin{array}{l}\text { Energy/Food } \\
\text { Intake }\end{array}$ & Weight & $\begin{array}{l}\text { VAT/ } \\
\text { SAT }\end{array}$ & $\begin{array}{c}\text { Glucose } \\
\text { (FG/GTT/ITT) }\end{array}$ & Hormones & HOMA-IR & Adipocytokine & Lipid Profile & Endotoxins \\
\hline 41 & $\begin{array}{l}\text { Vezza et al,, } \\
2019[77]\end{array}$ & $\begin{array}{l}\mathrm{C} 57 \mathrm{BL} / 6 \mathrm{~J} \\
\text { mice, } \mathrm{M}, \\
5 \mathrm{w}\end{array}$ & $\begin{array}{l}\text { 9/grp NDND + } \\
\text { OL HFDHFD + } \\
\text { LDHFD + } \\
\text { MDHFD + HD } \\
\text { FT* o only results } \\
\text { for LD, MD, } \\
\text { HD reported in } \\
\text { this review }\end{array}$ & $\mathrm{NA}$ & $5 \mathrm{w}$ & $60 \%$ & 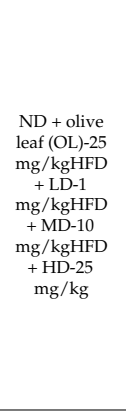 & $\begin{array}{l}\text { Oral } \\
\text { gav- } \\
\text { age }\end{array}$ & 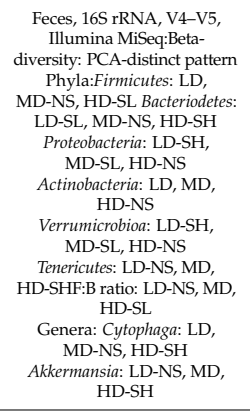 & $\begin{array}{l}\mathrm{LD}, \mathrm{MD}, \\
\mathrm{HD}-\mathrm{NS}\end{array}$ & $\begin{array}{l}\mathrm{LD}, \mathrm{MD}, \\
\mathrm{HD}-\mathrm{SL}\end{array}$ & $\begin{array}{l}\text { Epid:LD, } \\
\text { MD, HD-SL }\end{array}$ & $\begin{array}{l}\text { FG:LD, MD-NS, } \\
\text { HD-SL } \\
\text { OGTT(AUC):LD, } \\
\text { MD, HD-SL }\end{array}$ & $\begin{array}{l}\text { LD, MD, } \\
\text { HD-SL }\end{array}$ & $\begin{array}{l}\mathrm{LD}, \mathrm{MD}, \\
\mathrm{HD}-\mathrm{SL}\end{array}$ & & $\begin{array}{l}\text { LDL:LD-NS, } \\
\text { MD, HD-SL } \\
\text { HDL: } \\
\text { MD, HD-NS }\end{array}$ & \\
\hline 42 & $\begin{array}{l}\text { Henning } \\
\text { et al., 2018 } \\
{[78]}\end{array}$ & $\begin{array}{c}\text { C578DL/6J } \\
\text { mice (strain } \\
\text { JAX 00064), } \\
\text { M, 6-7 w w }\end{array}$ & $\begin{array}{c}\text { 12/grp } \\
\text { NDHFHSHFHS } \\
+ \text { GTPHFHS + } \\
\text { BTP }\end{array}$ & $p$ & $4 w$ & $\mathrm{NA}$ & $\begin{array}{c}0.5 \mathrm{~g} / 100 \mathrm{~g} \\
\text { of diet } \\
\text { providing } \\
\text { 0.25 } \\
\text { polyphe- } \\
\text { nolys/100 } \\
\text { diet of green } \\
\text { tea } \\
\text { polyphenol } \\
\text { (GTP) or } \\
\text { black tea } \\
\text { polyphenol } \\
\text { (BTP) }\end{array}$ & $\begin{array}{c}\text { Within } \\
\text { diet }\end{array}$ & 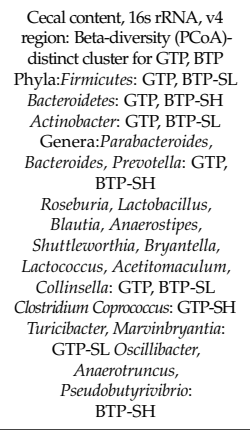 & $\begin{array}{l}\text { GTP-SL, } \\
\text { BTP-NS }\end{array}$ & $\begin{array}{c}\text { GTP, } \\
\text { BTP-SL }\end{array}$ & $\begin{array}{c}\text { Mesen:GTP, } \\
\text { BPT-SL, } \\
\text { Epidd GTP, } \\
\text { BTP-SLAT: } \\
\text { GTP, } \\
\text { BTP-NS }\end{array}$ & & & & & & \\
\hline \multicolumn{19}{|c|}{$\begin{array}{l}\text { PHENOLIC EXTRACTS-RATS } \\
\end{array}$} \\
\hline 43 & $\begin{array}{l}\text { R. Zhao } \\
\text { et al., 2019 } \\
{[770]}\end{array}$ & $\begin{array}{l}\text { Sprague- } \\
\text { Dawley rats, } \\
\text { M, (250-270 } \\
\text { g) }\end{array}$ & $\begin{array}{c}\text { 12/grp NDHFD } \\
\text { HFD +P } \\
\text { PPLHFD + } \\
\text { PPPH }\end{array}$ & $p$ & $12 \mathrm{w}$ & $45 \%$ & $\begin{array}{l}\text { PPPL-150 } \\
\text { mg/kg of } \\
\text { Pomegranate } \\
\text { polyphenols } \\
\text { (PPP) } \\
\text { PPPH-300 } \\
\mathrm{mg} / \mathrm{kg} \text { of } \\
\text { PPP }\end{array}$ & $\begin{array}{l}\text { Oral } \\
\text { gav- } \\
\text { age }\end{array}$ & 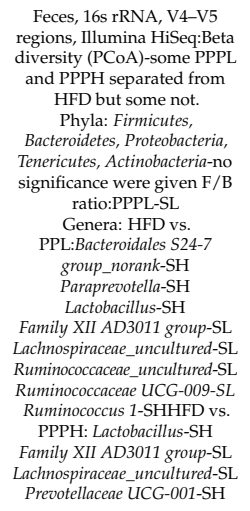 & $\begin{array}{l}\text { PPPL, } \\
\text { PPP-NS }\end{array}$ & $\begin{array}{c}\text { PPPL, } \\
\text { PPP-SL }\end{array}$ & & & & & $\begin{array}{l}\text { TNFa:PPPL, } \\
\text { PPPH-SL IL-6: } \\
\text { PPPL-NS, } \\
\text { PPPH-SL } \\
\text { IL-1B:PPPL-SL, } \\
\text { PPPH-NS }\end{array}$ & $\begin{array}{c}\text { TC:PPPL-SL, } \\
\text { PPPH-NS } \\
\text { TAG:PPPL- } \\
\text { NS, } \\
\text { PPPH-SL } \\
\text { HDL:PPPL, } \\
\text { PPPH-NS } \\
\text { LDL:PPPL, } \\
\text { PPH-SL }\end{array}$ & $\begin{array}{l}\text { LPS:PPPL- } \\
\text { SL, } \\
\text { PPPH-NS }\end{array}$ \\
\hline
\end{tabular}


Table 2. Cont.

\begin{tabular}{|c|c|c|c|c|c|c|c|c|c|c|c|c|c|c|c|c|c|c|}
\hline No. & $\begin{array}{l}\text { Author, } \mathrm{Yr}_{\mathrm{r}} \\
\text { [Referencel }\end{array}$ & $\begin{array}{c}\text { Species, } \\
\text { Sex, Age } \\
\text { (w)/ } \\
\text { Weight (g) }\end{array}$ & $\begin{array}{c}\text { Number of } \\
\text { Animals, } n l \\
\text { Groups (Grp) }\end{array}$ & $\begin{array}{l}\text { Prevention } \\
(p) / \text { Treatment } \\
\text { (T) }\end{array}$ & $\begin{array}{l}\text { Duration of } \\
\text { Intervention } \\
\text { (d/ws/mths) }\end{array}$ & $\begin{array}{l}\mathrm{HFD} \\
(\% \mathrm{Fat})\end{array}$ & Dosage & MOA & $\begin{array}{c}\text { Sample/Method/ } \\
\text { Hypervariable Region/GM } \\
\text { Composition }\end{array}$ & $\begin{array}{l}\text { Energy/Food } \\
\text { Intake }\end{array}$ & Weight & $\begin{array}{l}\text { VAT/ } \\
\text { SAT }\end{array}$ & $\begin{array}{c}\text { Glucose } \\
\text { (FG/GTT/ITT) }\end{array}$ & Hormones & HOMA-IR & Adipocytokine & Lipid Profile & Endotoxins \\
\hline 44 & $\begin{array}{l}\text { S. Lee et al,, } \\
2018[800]\end{array}$ & $\begin{array}{l}\text { Wistar rats, } \\
\mathrm{M}, \\
(200-220 \mathrm{~g})\end{array}$ & $\begin{array}{c}\text { 8/grp } \\
\text { NDHFDHFD + } \\
\text { BB }\end{array}$ & $p$ & $8 \mathrm{w}$ & $45 \%$ & $\begin{array}{l}\text { HFD with } 10 \\
g \\
\text { freeze-dried } \\
\text { blueberry } \\
\text { powder } \\
(\mathrm{BB}) / 100 \mathrm{~g}\end{array}$ & $\begin{array}{c}\text { Within } \\
\text { diet }\end{array}$ & 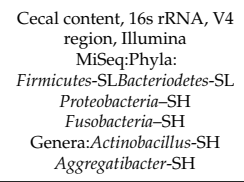 & & NS & VAT: NS & OGTT(AUC):NS & & & & & $\begin{array}{l}\text { Serum } \\
\text { LBP-SL }\end{array}$ \\
\hline 45 & $\begin{array}{l}\text { H. Xu et al., } \\
2019 \text { [81] }\end{array}$ & $\begin{array}{c}\text { Sprague- } \\
\text { Dawley rats, } \\
\text { M, } \\
5 \mathrm{w}\end{array}$ & $\begin{array}{c}\text { 6/grp } \\
\text { NDHFDHFD + } \\
\text { PFELHFD+ } \\
\text { PFEHHFD + } \\
\text { CAE(Positive } \\
\text { control * only } \\
\text { results for PFEL, } \\
\text { PFEH reported } \\
\text { in this review }\end{array}$ & $p$ & $8 w$ & $\begin{array}{c}\text { ND- } \\
76 \%+ \\
\text { fat- } \\
12 \%\end{array}$ & $\begin{array}{l}\text { PFEL-0.4\% } \\
\text { Pyracantha } \\
\text { fortuneana } \\
\text { extract (PFE) } \\
\text { PFEH-1\% } \\
\text { PFECAE- } \\
0.4 \% \text { Citrus } \\
\text { aurantium } \\
\text { extract } \\
\text { (CAE) }\end{array}$ & $\begin{array}{c}\text { Within } \\
\text { diet }\end{array}$ & 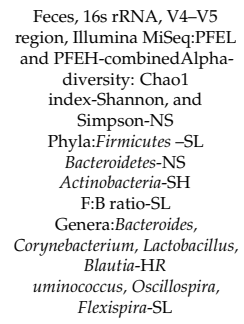 & $\begin{array}{l}\text { PFEL, } \\
\text { PFEH-NS }\end{array}$ & $\begin{array}{l}\text { PFEL, } \\
\text { PFEH-SL }\end{array}$ & $\begin{array}{l}\text { Epid/bw:PFEL- } \\
\text { NS } \\
\text { PFEH-SL }\end{array}$ & $\begin{array}{l}\text { FG:PFEL, } \\
\text { PFEH-SL }\end{array}$ & & & & 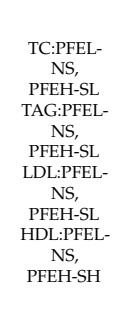 & \\
\hline
\end{tabular}

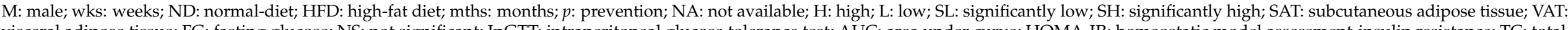

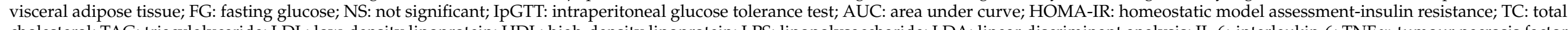

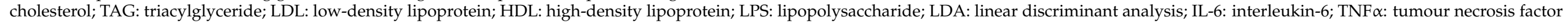

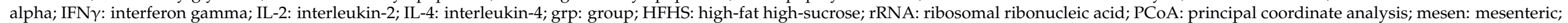

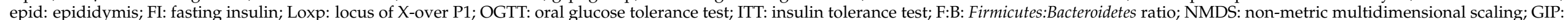

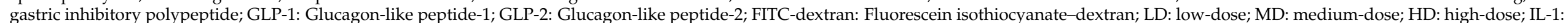

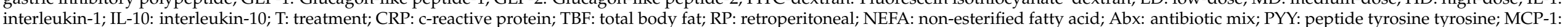

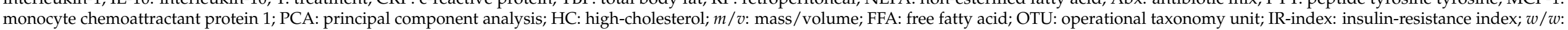
weight/weight; Il-1 $\beta$ : interleukin-1 beta; bw: body weight. 


\subsection{Study Characteristics}

Twenty-two studies used pure single phenolic compounds, while two studies used a combination of phenolic compounds. The remaining 22 studies investigated phenolic-rich extracts. Twenty-six studies assessed the effectiveness of flavonoids, and the remaining studies investigated stilbenes or phenolic acid or a combination of flavonoids and other polyphenol classes. Most studies $(n=38)$ used mice, while only seven used rats. All the studies included in this review used male animals. The study duration ranged from 2 to 24 weeks. A majority of the studies used either $60 \%$ HFD or $45 \%$ HFD. Thirty-three studies were based on a preventive model, i.e., the intervention and HFD intake were initiated simultaneously. Ten studies used the treatment model approach; i.e., treatment began a few weeks after HFD intake. This information was not clearly stated in two studies.

\subsection{Quality Assessment}

Risk of bias of included studies is summarised in Figure S2. Sequence generation, allocation of concealment, random outcome assessment and blinding (detection) were unclear in all the studies. Baseline characteristics were reported in $98 \%$ of the studies, and incomplete outcome data was low in $96 \%$ of the studies. The animals were randomly housed in $73 \%$ of the studies while this was not carried out in $27 \%$ of the studies. The study quality was also assessed using the GSP checklist, which provided a quantitative assessment of each study. As depicted in Figure S3, five studies scored above 13 with the highest score being 14 , while 40 studies scored between 7-12 with the lowest score being 9. Most studies did not address sample-size calculation, concealment of allocation, the animal's weight, time of intervention, method and time of sampling, number, and reason of excluded animals. The detailed GSPC scores are presented in Table S1.

\subsection{Effect of Polyphenols on Food/Energy Intake, Obesity-Related Parameters, and} Markers-Associated with Inflammation

The results are presented as significantly high (SH), significantly low (SL), or as not significant (NS) which refers to either a parameter being significantly increased, decreased or not-significant compared to HFD group. Studies that reported more than a compound or tested various dosages are presented as separate studies.

\subsubsection{Effect of Polyphenols on Energy/Food Intake and Bodyweight}

Thirty-two studies reported changes in energy/food intake, among which three studies that used pure phenolic compounds (PPC) $[35,40,42,43]$ and two $[68,78]$ studies that investigated phenolic extracts (PE) reported a significant drop in energy/food intake compared to HFD. Significantly higher food consumption was recorded in three studies [2PPC [41,50], 1-PE [73], while 24 studies reported non-significant difference in energy/food intake $[45-47,51,52,57,59,60,62,63,66,74,75,80]$ (Figure S4). As for bodyweight, thirty studies $(67 \%)$ recorded a significant drop post-treatment, of which 16 with PPC [35-45,50,52$54,56,57]$, and 15 with PE $[60,61,63,65,66,68,71-79,81]$. Non-significant observations were made in 19 studies [11-PPC [37,38,44,46-49,51,55,58], 9-PE [59,60,62,64,65,67,69,70,80]] (Figure S4).

\subsubsection{Effect of Polyphenols on Adiposity}

The adiposity in animal models was presented as the weight of visceral adipose tissue (VAT), subcutaneous adipose tissue (SAT) or total body fat (TBF). The VAT was significantly reduced in 24 studies [PPC-14 [37-39,41-44,46,48,50-54,57], PE-[61,63,65,68,71$73,77,78,81]]$, and 11 studies reported non-significance $[40,44,45,49,65,67,69,70,76,80,81]$. As for the SAT, seven studies showed a significant reduction [4-PPC [35,37,40,42,43], 3PE [61,62,73]], while 10 studies showed non-significant findings [36,38,39,44-46,48,49,51, $53-59,63,65,66,71,74,75,77,79-81]$. The TBF was significantly lowered in two studies that investigated PPC $[60,81]$ while three studies $[60,62,64]$ indicated non-significant changes (Figure S5). 


\subsubsection{Effect of Polyphenols on Lipid Profile}

Total cholesterol was significantly lowered in 11 studies following the administration of PPC [37,38,41-43,46,50,52,54,56-58] and eight studies for PE [63,68,71,73-76,79,81]. One study reported a significant increase with PPC [45]. Thirteen studies reported nonsignificant findings $[35,36,38,46,49,55,56,66,67,71,72,79,81]$. As for TAG, PPC significantly lowered the index in 12 studies [35,37,39,41-44,50,52,54,55,57,58], and PE in 11 studies $[60,61,63,65,68,70,72,73,76,79,81]$. Nine studies that used PPC significantly lowered LDL $[36,38,44,46,52,54,56-58]$ level and eight studies showed a similar result with PE [63, $65,66,71,76,77,79,81]$. As for the HDL level, four studies recorded a significant reduction [3PPC [38,46,56], 1-PE [63]], and five studies a significant increment [5-PPC [44,45,52,54,55], 2-PE [66,81]] (Figure S6).

\subsubsection{Effect of Polyphenols on Glucose Homeostasis}

The fasting glucose (FG) was reported in 24 studies, from which nine studies reported a significant reduction following administration of PPC [37-39,41,42,44,48,55,57] and eight for PE [63-65,72,74-77,81]. Twelve studies reported non-significant changes in FG [35, $38,50,55,62,64-66,68,70,73,77]$. The oral/intraperitoneal glucose tolerance test (GTT) was evaluated in 21 studies, and among these, five PPC [41-43,47,53,57] and nine PE [59$62,64,67,72,76,77]$ showed significant improvement in glucose tolerance, and 12 studies reported non-significant changes $[48,51,55,59,60,62,64,67-70,80]$. Fasting insulin (FI) was significantly lowered in four studies with the use of PPC $[39,42,43,48,54]$ and six studies with PE $[65,66,68,70,72,77]$. Non-significant observations were recorded in nine studies [55, $57,62,65-67,70,73,80]$. HOMA-IR was significantly lowered in 13 studies [4-PPC [39,48,55, 57], 9-PE [60,65-68,72-75,77]], and five studies recorded non-significant changes [55,60,6567] (Figure S7).

\subsubsection{Effect of Polyphenols on Adipocytokines, CRP, and LPS/LBP}

Thirteen 13 studies investigated TNF $\alpha$ level in serum/plasma, from which 12 studies recorded a significant reduction $[36,37,41,44,46,51,54,61,66,71,74,75,79]$ and two studies recorded non-significant changes [37,44]. As for IL-6, 11 studies showed a significant reduction $[36,37,39,46,51,54,61,66,71,74,79]$, while two studies reported non-significant changes $[37,79]$. MCP-1 was significantly lowered in four studies $[51,54,60,74,75]$, and two studies $[60,74,75]$ recorded non-significant observations. Meanwhile, leptin was significantly reduced in three studies [36,42,43,61], and two studies recorded non-significant findings $[54,67]$. Adiponectin was only evaluated in two studies, of which one showed a significant increment [54], and another recorded a non-significance [42,43]. The CRP level was significantly lowered in two studies [46,71]. The LPS/LPB was significantly reduced in 12 studies that investigated PPC [35-37,39,41-44,46,48,50,56,57] and 11 studies that investigated PE $[60,61,63,66,68,70,71,73-75,79,80]$. Six studies recorded non-significant changes in LPS/LBP concentrations [37,60,62,66,70,79] (Figure S8).

\subsubsection{The Overall Effect of Polyphenols on Obesity-Related Parameters and Inflammation}

Figure 2 summarises the overall impact of the phenolic intervention on various obesityrelated parameters and inflammation. The most profound effects $(83 \%)$ were seen on either body weight or visceral adiposity or TAG or all three $[35-48,50-54,56-58,60,61,63,65,66,68$, $70-79,81]$. While $13 \%$ of the study saw improvement only in glucose parameters $[55,59,62$, $64,67,69]$, and $2 \%$ (one study) of the study reported improvement in inflammatory markers and LPS [80]. The remaining $2 \%$ (one study) of the study did not significantly improve the metabolic parameters measured [49]. 


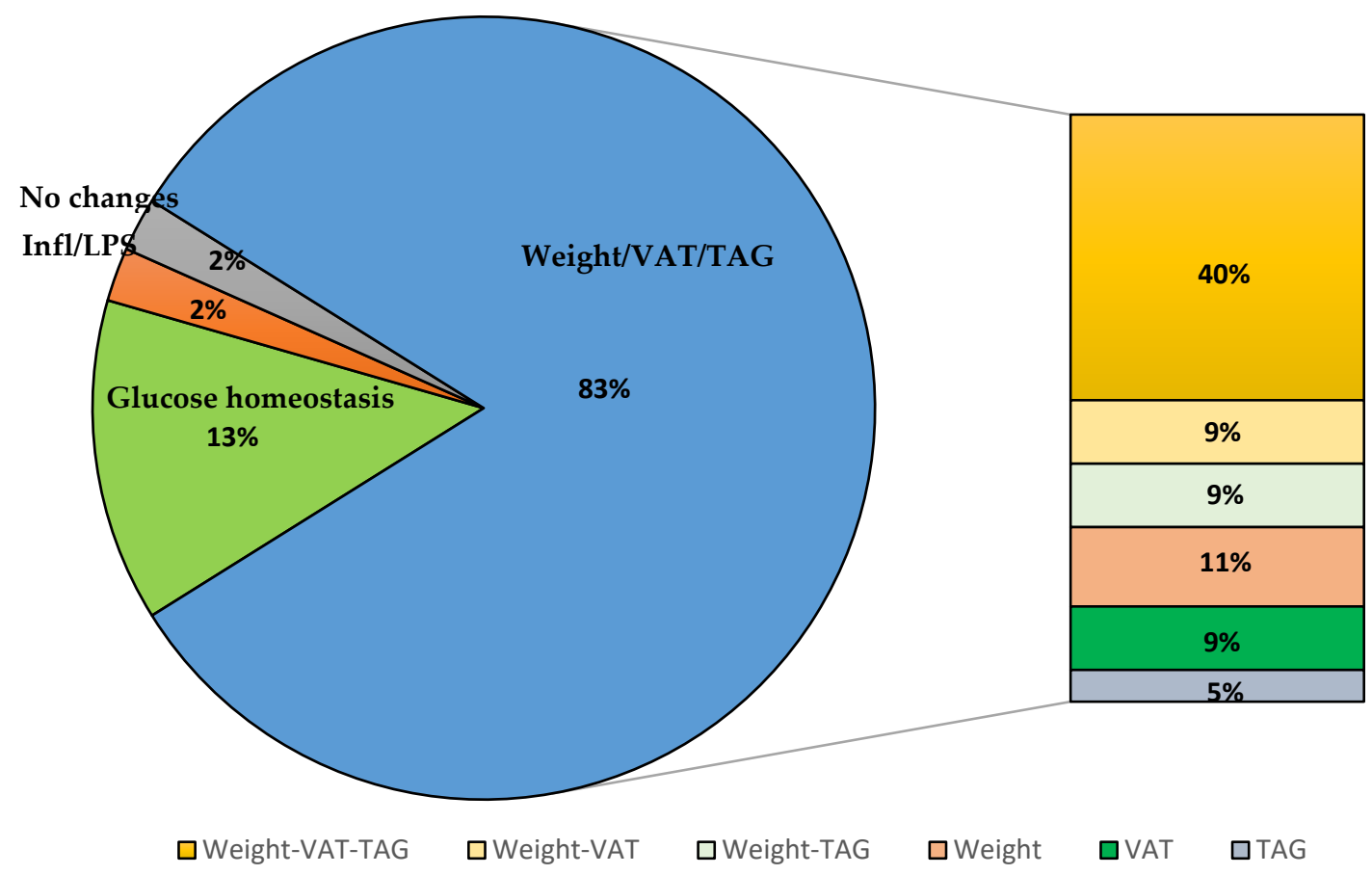

Figure 2. Overall effect of the phenolic intervention on metabolic parameters. VAT-visceral adipose tissue, TAGtriacylglyceride, Inf-inflammatory markers, LPS-lipopolysaccharide.

\subsection{Effect of Polyphenols on Gut Microbiota}

We report the changes in GM as alpha diversity, beta-diversity, Firmicutes: Bacteroidetes ratio (F:B ratio), overall changes in phyla, family/genus and frequently modulated genera.

\subsubsection{Alpha and Beta-Diversity}

The included studies' alpha-diversity were reported as ACE, Chao1, OTU, Shannon, Simpson, Inverse Simpson or PD. The alpha-diversity was significantly increased in 10 studies [4-PPC [41,54,55,57], 6-PE [60,61,63,71,73,76]], while six studies recorded a significant decrease [4-PPC [44,46,49,53], 2-PE [65,71]]. Non-significant observations were recorded in 17 studies [10-PPC [35,40,41,44-46,48,49,51,52], 7-PE [59,60,65,71,72,74,75,81]] (Table S2). The beta-diversity was based on the PCoA/PCA/NMDS plots. Among the studies which tested PPC, 12 studies showed improvement in beta-diversity [36,37,41-43,45-47,49,51,5355], and 14 studies for PE [59,61-63,65,68-73,76-78], i.e., there were formations of clusters near normal-diet fed rats or formations of distinct cluster away from normal or high-fat-fed animals. Nine studies showed no improvement [PPC [39,40,44,46,48,56,57], PE [60,70]], i.e., there were formations of clusters with HFD/some overlapping with HFD (Figure S9).

\subsubsection{Modulation of Firmicutes:Bacteroidetes Ratio (F:B Ratio), Phyla, and Family/Genus}

As presented in Figure 3, F:B ratio was significantly lowered in nine studies that tested PPC [37,39,42,43,45,47,49,55-57] and seven studies for PE $[61,69,71,72,77,79,81]$. Nonsignificance was recorded in 17 studies [9-PPC [36,37,44,46,48,52,54,55,58], 8-PE [59,62,64, $71,73-75,77,79]]$. At the phyla level, significant changes were reported in 24 studies [36,37, $39-41,45,46,49-51,54-57,62,66,68,70,71,76-78,80,81]$, while 42 studies showed a significant modulation of either family/genus [36,37,39-73,76-83]. Eleven studies did not observe any significance in phyla $[48,52,53,58,59,62,64,65,67,72,73]$. Three studies did not perform any statistical analysis $[35,38,74,75]$. 


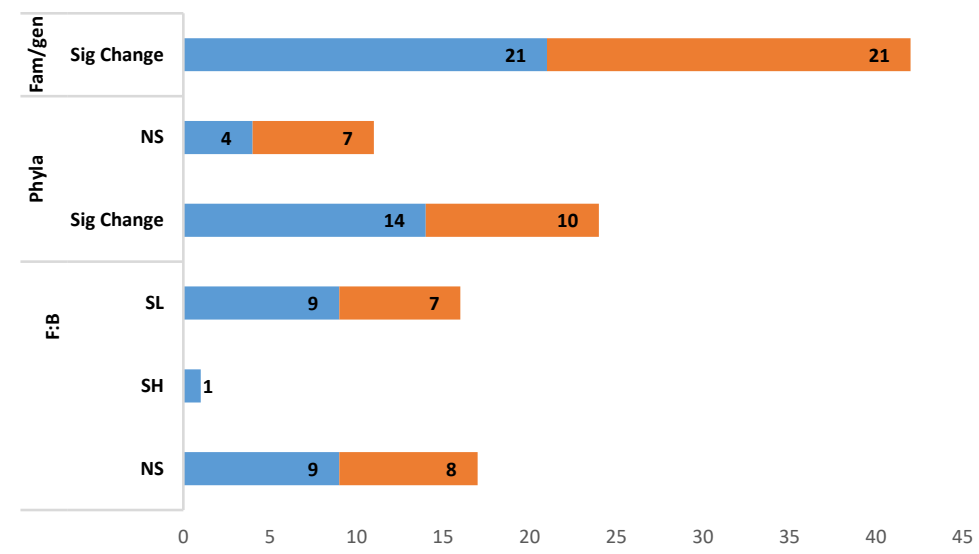

Figure 3. Effect of polyphenols on Firmicutes:Bacteroidetes ratio, phyla, and family/genus. SLSignificantly Low, SH-Significantly High, Sig change-significant changes, NS-Not Significant, Famfamily, gen-genus. Significance/non-significance compared to HFD. Studies that tested more than one compound/dose: $[45,54,57,58,62,63]$.

\subsubsection{Frequently Modulated Gut Microbes}

Figure 4 explains the most frequently modulated gut microbes following the phenolic interventions. The most notable improvement was observed for Akkermansia, whereby 12 studies reported a significant increase [six-PPC [37,41-43,46,49,57], six-PE [61,66,68,70,73, 77]], while two studies observed a significant reduction $[47,76]$. Bacteroides was significantly increased in six studies $[37,47,59,66,71,78]$, and three reported a significant reduction $[51,58$, 76]. Blautia and Roseburia were upregulated in six studies [51,55,62-67] and downregulated in two [56,58,78,79]. Bifidobacterium was significantly high in four studies [one-PPC [35], three-PE [61,66,73]] and lowered in two studies [37,70], Lactobacillus was significantly high in three studies $[48,76,79]$ and significantly low in six studies $[56,57,68,70,71,78]$. Alistipes was significantly increased in three studies [57,66,71] and significantly reduced in one [76]. Desulfovibrio was significantly increased in one study [64] and decreased in three studies $[39,61,67]$.

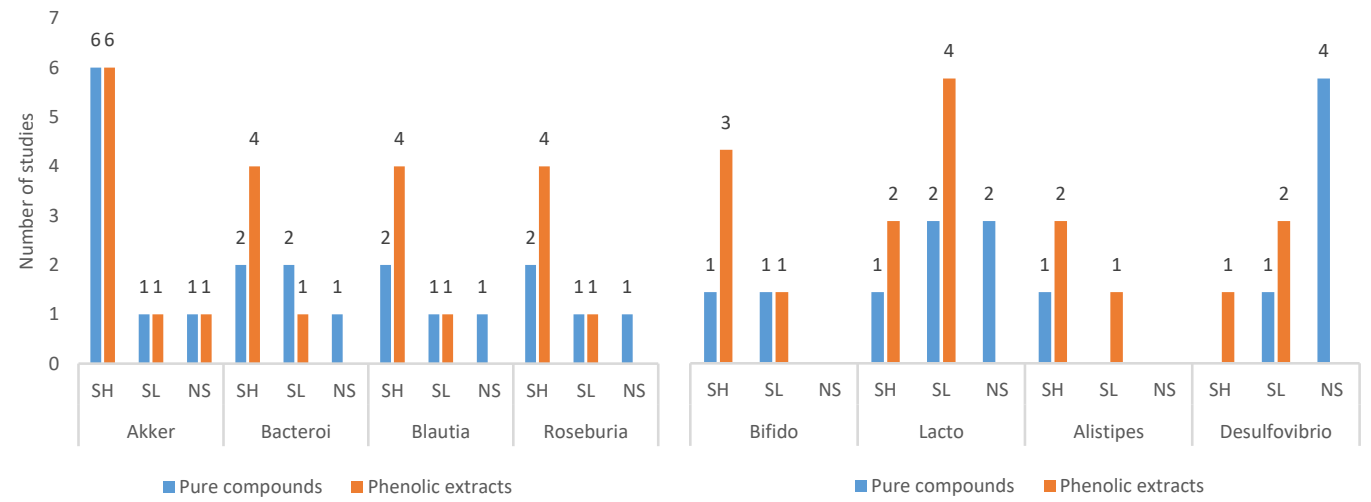

Figure 4. Frequently modulated gut microbes. Akker-Akkermansia, Bacteroi-Bacteroides, Bifi-doBifidobacterium, Lacto-Lactobacillus, SL-Significantly Low, SH-Significantly High. NS-Not Significant. Significance/non-significance compared to HFD.

\section{Discussion}

Polyphenols are emerging as prebiotics due to their ability to improve intestinal dysbiosis and health parameters [29,30]. Numerous preclinical studies have investigated the role of polyphenols in improving various health conditions in animal models. This comprehensive review was carried to obtain a complete and exhaustive summary of the preclinical studies investigating the effect polyphenols in ameliorating HFD-induced obesity. 
In general, $98 \%$ of the studies reviewed indicated significant improvement in the metabolic derangements inflicted by HFD intake after consuming pure phenolics compounds or its extracts. These changes were related to the modulation of GM at the genus level. Our analysis also indicates that all 23 studies that tested pure PP and 21 out of 22 studies used extracts observed improvement in metabolic parameters and gut microbiota profiles. Thus, we can conclude that both pure PP and extracts were equally effective as prebiotics. However, this review's findings will need to be interpreted with some caution as the quality score for the majority of studies was below 12 . This was because a few essential criteria such as sequence generation, allocation of concealment, random outcome assessment, blinding (detection), and sample size calculation were not addressed in most studies.

\subsection{Effect of Polyphenols on Obesity-Associated Parameters, Adipocytokines, and LPS/LBP}

It is evident that phenolic intervention significantly reduced one or more metabolic parameters such as weight gain, VAT, FBG, FI, GTT, HOMA-IR, TC, TAG, LDL, LPS, TNF $\alpha$ and IL- 6 in $98 \%$ of the studies included in this review.

The most prominent observation in this review was the reduction in body weight/VAT/ TAG reported in $83 \%$ of the studies. Among these, $40 \%$ of studies showed a decrease in all three parameters, i.e., body weight, VAT and TAG. Therefore, it can be assumed that the reduction in body weight is probably related to reduced VAT and TAG. The mechanisms highlighted for TAG improvement in some of these studies include downregulation of $A g-$ pat2 [60], reduction in taurine-conjugated bile acids causing activation of nuclear farnesoid $X$ receptor (FXR) in the liver, consequently, suppressing lipogenic pathway in the liver [61], inhibition of hepatic adipogenic genes such as Fas, PPAR $\gamma$, and SREBP-1c [71,72], and upregulation of fasting-induced adipose factor (FIAF) expression in the intestine, hence, suppressing TAG deposition [81]. Our observation indicates that the mode of action varies between different PP.

\subsection{Effect of Polyphenols on Glucose Homeostasis}

We also observed that studies reporting a reduction in weight, VAT, and or TAG revealed a significant improvement in glucose homeostasis (either FBG, GTT, FI or HOMAIR), and its inflammatory state. However, whether the improvement in glucose homeostasis is a cause or effect of a decreased inflammatory state is unclear. Although many studies have hypothesised that low-grade systemic inflammation in white adipose tissue disrupts glucose homeostasis, a clear mechanism of HFD-induced hyperglycaemia or IR remains elusive [84-86]. Few other studies mention that IR precedes WAT inflammation. Shimobayashi et al. [87] reported that the accumulation of macrophages in WAT preceded IR in HFD and mTORC2-knockout animal models. They further stated that IR in WAT induced MCP-1 production responsible for the recruitment of monocytes and activation of macrophages in WAT. Lee et al. [88], on the other hand, observed IR and glucose intolerance as early as day 3 following HFD intake, and this was associated with lipid overload in the adipose tissue. Chronic inflammation was identified as a critical mechanism for IR once obesity is established. Apart from this, ellagic acid and its metabolites were demonstrated to mitigate IRs by improving mitochondrial function by activating 5'-AMP-activated protein kinase [89-91]. Some PPs such as geraniin, corilagin, and ellagic acid are also shown to exhibit insulin-like activity, causing 2-NBDG uptake in the absence of insulin by preadipocytes [92].

Few PPs only improved glucose intolerance/IR without affecting weight/VAT/TAG. The exact mechanisms for this were not well elaborated in the papers included in this review. Nevertheless, the mechanism of actions was discussed extensively in a few reviews [14,93-95]. Some of which include; inhibition of salivary and pancreatic $\alpha$-amylase and $\alpha$-glucosidase activities. These enzymes are responsible for metabolising carbohydrate and its inhibition of reduced carbohydrate metabolism and therefore, glycaemic spike [14,94]. This effect has been reported with punicalagin [96], ferulic acid, and isofer- 
ulic acid ( $\alpha$-glucosidase inhibitors) [97]. Certain polyphenols were also shown to inhibit glucose transporters in the gut, therefore reducing glucose uptake. For instance, quercetin, chamomile and green tea were shown to inhibit glucose uptake in the intestine by inhibiting GLUT2, and GLUT5 [98,99]. Some polyphenols such as isovanillic acid 3-O-sulfate (metabolites of cyanidin 3-O-glucoside) [100] and epicatechin [101] have been shown to increase glucose intake by upregulating GLUT4 expression in tissues.

\subsection{Effect of Polyphenols on Gut Microbiota}

Approximately $93 \%$ of the studies included in this review reported significant changes in one or more parameters of GM modulation. It is unclear whether the remaining $7 \%$ of the studies (three studies) performed any statistical analysis to prove GM modulation and only reported their findings in relative abundance changes. Nevertheless, these studies did report positive changes with phenolic interventions.

The alpha-diversity was reported in 29 studies (64\%); among these nine studies reported a significant increase in this diversity. Beta-diversity was presented as a PCOA plot in most studies. Among the 35 studies that reported this diversity, 26 plots showed distinct cluster/clustering closer to normal diet groups. The phenolic intervention did not improve alpha-diversity in most studies, which could be due to the shorter study duration [102]. Furthermore, diet-diversity was previously observed to affect beta and not alpha-diversity [103].

F:B ratio was reported in 28 studies, with 17 studies indicating a significant reduction of the ratio. This raises the question of phyla level relevancy often reported in obesity studies. Higher firmicutes and lower bacteroidetes (high F:B ratio) are often associated with obesity [104-106]. On the contrary, few studies showed a low F:B ratio [107-109]. A few meta-analyses have also shown a lack of differences in F:B ratio between obese and lean individuals [110-112]. It was suggested that the F:B ratio may not be a robust indicator of dysbiosis in obesity [113]. It was further highlighted that the disparities that exist between studies could be related to methodological differences such as sample collection, DNA extraction, hypervariable regions of interest, sequencing methods and bioinformatics data analysis software (QIIME, Mothur) [113].

Nevertheless, polyphenols significantly improved relative abundance of GM at the family/genus level in 42 studies. A few genera were consistently observed to be modulated irrespective of the type of intervention. The commonly improved genera include Akkermansia, Bacteroides, Blautia, and Roseburia, and to a lesser extent, Bifidobacteria, Lactobacillus, and Alistipes. Desulfovibrio, on the other hand, was reduced in three studies. The genus Akkermansia was significantly increased with the intake of vanillin, anthocyanin, kudingcha dicaffeoylquinic acids, EGCG, quercetin-resveratrol, total flavonoid, blueberry extract, tea polyphenols, cranberry extracts, cloudberry extract, alpine bearberry extract, green tea extract, and olive leaf extract. Akkermansia muciniphila (A. muciniphila), is a gramnegative bacterium belonging to the Verrucomicrobia phylum. This bacterium produces mucin degrading enzymes causing fermentation of mucin to acetic, propionic acid, and sulphate [114]. A preclinical study reported that A. muciniphila reduced plasma TAG, improved fatty liver, and gut dysbiosis [115]. In a randomised human trial, administration of A. muciniphila for 30 days improved plasma insulin, HOMA-IR, Dipeptidyl-peptidase 4 (DPP4) (inflammation) and LPS concentration in overweight/obese individuals [116]. Administration of procyanidins, resveratrol, Rubus occidentalis, tea polyphenols, kudingcha tea, fuzhuan brick tea, green tea polyphenol, and black tea polyphenol improved Bacteroides. Some Bacteroides strains were categorized as next-generation probiotics [117], including B. uniformis, B. acidifaciens, and B. dorei. These strains were shown to ameliorate HFD-induced metabolic derangements [117].

In contrast, some strains of $B$. fragilis were identified as a risk factor for the development of colon cancer [118]. It can therefore be concluded that the health benefit of the genus is strain specific. Blautia was improved with grape seed proanthocyanidin extract, resveratrol, Rutgers scarlet lettuce, green tea, oolong tea and black tea, rapeseed oil with sinapine, 
tea polyphenols. This genus is a butyric producer, reported to ameliorate intestinal inflammation, IR [119], and exhibited an inverse association with visceral adiposity [120]. Therefore, it may play a crucial role in the management of obesity and obesity-related diseases. Roseburia was improved with grape seed proanthocyanidin extract, a combination of resveratrol and sinapic acid, Rutgers scarlet lettuce, grape seed extract, tea polyphenols, grape pomace extract. Roseburia also a butyrate producer and this genus has been implicated in the control of intestinal inflammation and was shown to be reduced in patients with type II diabetes [121]. The conventional probiotics such as Bifidobacterium and Lactobacillus were improved in fewer studies. Administration of kudingcha dicaffeoylquinic acids, blueberry extract, and tea polyphenols improved Bifidobacterium, while Lactobacillus was improved with the intake of hydroxytyrosol, pandanus tectorius fruit extract, pomegranate polyphenols. Some of the health benefits of Bifidobacterium include improvement in celiac disease with the administration of $B$. infantis strain NLS [122], irritable bowel syndrome with B. lactis DN-173010 [123]. Conversely, Lactobacillus was significantly improved in three studies, while six studies showed a significant reduction.Since only the changes up to genus level were reported in all the studies, it is uncertain the type of species/strains affected following phenolic interventions. Literature suggests that the health benefits of Lactobacillus are species/strain-dependent. For instance, weight loss was reported with L. gasseri [124], and L. reuteri JBD301 [125]. Whilst, weight gain was reported with L. acidophilus [126]. The abundance of Alistipes improved with the administration of total flavonoids of quzhou, tea polyphenols, kudingcha and fuzhuan brick tea. Similar to Lactobacillus, the health attributes of this genus are species/strain specific. Among patients with liver cirrhosis, lower A. shahii and A. putredinis levels were observed compared to healthy controls [127], while $A$. finegoldii was shown to promote colorectal cancer [128]. Desulfovibrio is known to produce a cytotoxic compound; hydrogen sulfide. This bacterium was observed to increase in patients with ulcerative colitis [129].

\subsection{Is LPS the Only Linking Factor between Metabolic Derangements and Gut Dysbiosis?}

Consumption of HFD was proven to increase fat deposition, elicit IR, and inflammation in adipose tissue, liver and skeletal muscle $[130,131]$. LPS was discovered as one of the linking factors between HFD intake and metabolic derangements. The administration of LPS into lean mice on normal-diet caused similar changes as the HFD-fed mice [130]. Therefore, we explored the relationship between GM, LPS, and metabolic parameters. Among the 23 studies that reported a significant reduction in LPS/LBP, 20 stated significant modulation in GM and metabolic derangements following PP administration. Two studies [35,74] reported significant improvement in metabolic parameters and LPS concentration; however, they did not indicate GM's statistical significance. Thus, the relationship between GM modulation and LPS is unclear for these two studies. Interestingly, Cheng et al., 2016 [62], reported non-significance for LPS even though GM modulation and significant improvement in OGTT were reported with Rutgers scarlet lettuce. This suggests that other factors may also play a role in the amelioration of metabolic derangements such as bile acids, and short-chain fatty acids (SCFA). Gut microbiota composition has been shown to alter the type of secondary bile acids, and consequently influence glucose homeostasis [132]. It has also been shown to improve SCFA production; for instance, the administration of butyrate was shown to improve rodent's insulin sensitivity [133]. Considering the studies included in this review, we conclude that PP's administration improved GM composition and consequently, the metabolic parameters. This was achieved partly by reducing LPS concentration; however, the involvement of other mechanisms cannot be discounted.

\section{Limitations}

In general, PP interventions significantly improved HFD-induced metabolic derangements in $98 \%$ of the animal studies and these changes were shown to be related to the GM modulation. Nevertheless, there are some limitations to this review, which will be discussed here. 


\subsection{Experimental Animals and Their Environment}

A variety of experimental animal species (C57BL/6J, C57BL/6N, C57BL/6 wild type Sprague Dawley rats, Wistar rats) of varying ages were used. These experimental animals were exposed to different types of a high-fat diet, water source, bedding materials, and were either housed alone or in groups.

\subsection{Types of Intervention}

Different types of phenolic compounds were tested, either in its pure form or as an extract at varying doses. These compounds were also administered via various modes (gavage/within diet or drinking water).

\subsection{Methodology to Assess GM}

GM modulation may have been affected due to variations in the methodology employed between studies, including the use of faecal samples or intestinal contents, faecal sampling time, and the difference in the hypervariable regions, sequencing methods, and bioinformatics tools utilised.

\subsection{Outcomes Reported}

The parameters reported for metabolic derangements and GM modulation differed, i.e., not all studies reported the same parameters. These variations greatly influenced the reported outcomes, thus posing a challenge in summarising polyphenols' prebiotic effect in preclinical studies.

\section{Conclusions}

Polyphenols appear to be a promising phytochemical in the management of dietinduced obesity. This review, havedemonstrated that the administration of polyphenols to obese animals improved metabolic derangements, particularly, weight gain, visceral adiposity, plasma TAG, and glucose homeostasis inflicted by HFD intake. These improvements were established to be the result of improved gut dysbiosis as presented in all the studies. The findings can form the basis for the development of randomised clinical trials required to conclusively place polyphenols as prebiotics. Moreover, Nonetheless, discrepancies in study designs, metabolic markers measured, and the methodology of gut microbiota analysis in preclinical studies need to be streamlined to make a more robust conclusion.

Supplementary Materials: The following are available online at https://www.mdpi.com/2304-8 158/10/2/299/s1, Figure S1. Search strategy (PubMed); Figure S2. Risk of bias using SYRCLE's Risk of Bias tool; Figure S3. Study quality using Gold Standard Publication Checklist, Figure S4. Effect of polyphenols on energy/food intake, and body weight; Figure S5. Effect of polyphenols on adiposity; Figure S6. Effect of polyphenols on lipid profile; Figure S7. Effect of polyphenols of glucose homeostasis; Figure S8. Adipocytokines, C-Reactive Protein (CRP), and LPS/LBP; Figure S9. Beta-diversity of (a) pure phenolic compounds and (b) phenolic extracts; Table S1. Gold Standard Publication Checklist of study quality; Table S2. Alpha diversity of pure phenolic compounds and extracts.

Author Contributions: Conceptualization, M.M., and U.D.P.; Methodology, M.M., U.S., and U.D.P.; Writing-Original Draft Writing, M.M.; Review and Editing, U.S., and U.D.P. Supervision, U.D.P. Funding Acquisition, U.D.P. All authors have read and agreed to the published version of the manuscript.

Funding: This work was funded by the Ministry of Higher Education, FRGS/1/2017/SKK08/MUSM/ 02/2, and Tropical Medicine and Biology (TMB) Platform, Monash University. The APC was funded by Jeffrey Cheah School of Medicine, Monash University.

Conflicts of Interest: The authors declare no conflict of interest. 


\section{References}

1. CDC. The Health Effects of Overweight and Obesity 2020. Available online: https://www.cdc.gov/healthyweight/effects/index. html (accessed on 11 March 2020).

2. Pereira, M.A. Diet beverages and the risk of obesity, diabetes, and cardiovascular disease: A review of the evidence. Nutr. Rev. 2013, 71, 433-440. [CrossRef] [PubMed]

3. Kovesdy, C.P.; Furth, S.L.; Zoccali, C. Committee obotWKDS. Obesity and Kidney Disease: Hidden Consequences of the Epidemic. Am. J. Hypertens. 2017, 30, 328-336. [CrossRef] [PubMed]

4. Dye, L.; Boyle, N.; Champ, C.; Lawton, C. The relationship between obesity and cognitive health and decline. Proc. Nutr. Soc. 2017, 76, 443-454. [CrossRef] [PubMed]

5. Dai, H.; Alsalhe, T.A.; Chalghaf, N.; Riccò, M.; Bragazzi, N.L.; Wu, J. The global burden of disease attributable to high body mass index in 195 countries and territories, 1990-2017: An analysis of the Global Burden of Disease Study. PLoS Med. 2020, 17, e1003198. [CrossRef]

6. Schnurr, T.M.; Jakupović, H.; Carrasquilla, G.D.; Ängquist, L.; Grarup, N.; Sørensen, T.I.A.; Tjønneland, A.; Overvad, K.; Pedersen, O.; Hansen, T.; et al. Obesity, unfavourable lifestyle and genetic risk of type 2 diabetes: A case-cohort study. Diabetologia 2020, 63, 1324-1332. [CrossRef]

7. Ghosh, S.; Bouchard, C. Convergence between biological, behavioural and genetic determinants of obesity. Nat. Rev. Genet. 2017, 18, 731-748. [CrossRef]

8. $\quad$ Ridaura, V.K.; Faith, J.J.; Rey, F.E.; Cheng, J.; Duncan, A.E.; Kau, A.L.; Griffin, N.W.; Lombard, V.; Henrissat, B.; Bain, J.R.; et al. Gut Microbiota from Twins Discordant for Obesity Modulate Metabolism in Mice. Science 2013, 341, 1241214. [CrossRef]

9. Gérard, P. Gut microbiota and obesity. Cell. Mol. Life Sci. 2016, 73, 147-162. [CrossRef]

10. Cox, A.J.; West, N.P.; Cripps, A.W. Obesity, inflammation, and the gut microbiota. Lancet Diabetes Endocrinol. 2015, 3, 207-215. [CrossRef]

11. Bäckhed, F.; Ding, H.; Wang, T.; Hooper, L.V.; Koh, G.Y.; Nagy, A.; Semenkovich, C.F.; Gordon, J.I. The gut microbiota as an environmental factor that regulates fat storage. Proc. Natl. Acad. Sci. USA 2004, 101, 15718-15723. [CrossRef]

12. Turnbaugh, P.J.; Ley, R.E.; Mahowald, M.A.; Magrini, V.; Mardis, E.R.; Gordon, J.I. An obesity-associated gut microbiome with increased capacity for energy harvest. Nat. Cell Biol. 2006, 444, 1027-1031. [CrossRef] [PubMed]

13. Bäckhed, F.; Manchester, J.K.; Semenkovich, C.F.; Gordon, J.I. Mechanisms underlying the resistance to diet-induced obesity in germ-free mice. Proc. Natl. Acad. Sci. USA 2007, 104, 979-984. [CrossRef] [PubMed]

14. Kim, Y.; Keogh, J.B.; Clifton, P.M. Polyphenols and Glycemic Control. Nutrients 2016, 8, 17. [CrossRef] [PubMed]

15. Zhang, H.; Tsao, R. Dietary polyphenols, oxidative stress and antioxidant and anti-inflammatory effects. Curr. Opin. Food Sci. 2016, 8, 33-42. [CrossRef]

16. Justino, A.B.; Pereira, M.N.; Peixoto, L.G.; Vilela, D.D.; Caixeta, D.C.; De Souza, A.V.; Teixeira, R.R.; Silva, H.C.G.; de Moura, F.B.R.; Moraes, I.B.; et al. Hepatoprotective Properties of a Polyphenol-Enriched Fraction fromAnnona crassifloraMart. Fruit Peel against Diabetes-Induced Oxidative and Nitrosative Stress. J. Agric. Food Chem. 2017, 65, 4428-4438. [CrossRef] [PubMed]

17. Ajiboye, B.O.; Ojo, O.A.; Akuboh, O.S.; Abiola, O.M.; Idowu, O.; Amuzat, A.O. Anti-Hyperglycemic and Anti-Inflammatory Activities of Polyphenolic-Rich Extract of Syzygium cumini Linn Leaves in Alloxan-Induced Diabetic Rats. J. Evid. Based Integr. Med. 2018, 23. [CrossRef]

18. Palanisamy, U.D.; Ling, L.T.; Manaharan, T.; Appleton, D. Rapid isolation of geraniin from Nephelium lappaceum rind waste and its anti-hyperglycemic activity. Food Chem. 2011, 127, 21-27. [CrossRef]

19. Khan, H.; Reale, M.; Ullah, H.; Sureda, A.; Tejada, S.; Wang, Y.; Zhang, Z.J.; Xiao, J. Anti-cancer effects of polyphenols via targeting p53 signaling pathway: Updates and future directions. Biotechnol. Adv. 2020, 38, 107385. [CrossRef] [PubMed]

20. Choi, D.-Y.; Lee, Y.-J.; Hong, J.T.; Lee, H.-J. Antioxidant properties of natural polyphenols and their therapeutic potentials for Alzheimer's disease. Brain Res. Bull. 2012, 87, 144-153. [CrossRef] [PubMed]

21. Jiang, H.; Wang, J.; Rogers, J.; Xie, J. Brain Iron Metabolism Dysfunction in Parkinson's Disease. Mol. Neurobiol. 2016, 54, 3078-3101. [CrossRef]

22. Serino, A.; Salazar, G. Protective Role of Polyphenols against Vascular Inflammation, Aging and Cardiovascular Disease. Nutrients 2018, 11, 53. [CrossRef] [PubMed]

23. Pešić, M.B.; Milinčić, D.D.; Kostić, A.Ž.; Stanisavljević, N.S.; Vukotić, G.N.; Kojić, M.O.; Gašić, U.M.; Barać, M.B.; Stanojević, S.P.; Popović, D.A.; et al. In vitro digestion of meat- and cereal-based food matrix enriched with grape extracts: How are polyphenol composition, bioaccessibility and antioxidant activity affected? Food Chem. 2019, 284, 28-44. [CrossRef] [PubMed]

24. Efenberger-Szmechtyk, M.; Nowak, A.; Czyzowska, A. Plant extracts rich in polyphenols: Antibacterial agents and natural preservatives for meat and meat products. Crit. Rev. Food Sci. Nutr. 2020, 61, 1-30. [CrossRef] [PubMed]

25. Galanakis, C.M. Phenols recovered from olive mill wastewater as additives in meat products. Trends Food Sci. Technol. 2018, 79, 98-105. [CrossRef]

26. Fahmy, H.; Hegazi, N.M.; El-Shamy, S.; Farag, M.A. Pomegranate juice as a functional food: A comprehensive review of its polyphenols, therapeutic merits, and recent patents. Food Funct. 2020, 11, 5768-5781. [CrossRef] [PubMed]

27. Kumar Singh, A.; Cabral, C.; Kumar, R.; Ganguly, R.; Kumar Rana, H.; Gupta, A.; Lauro, M.R.; Carbone, C.; Reis, F.; Pandey, A.K. Beneficial effects of dietary polyphenols on gut microbiota and strategies to improve delivery efficiency. Nutrients 2019, 11, 2216. [CrossRef] [PubMed] 
28. Cardona, F.; Lacueva, M.C.A.; Tulipani, S.; Tinahones, F.J.; Queipo-Ortuño, M.I. Benefits of polyphenols on gut microbiota and implications in human health. J. Nutr. Biochem. 2013, 24, 1415-1422. [CrossRef] [PubMed]

29. Gibson, G.R.; Hutkins, R.; Sanders, M.E.; Prescott, S.L.; Reimer, R.A.; Salminen, S.J.; Scott, K.; Stanton, C.; Swanson, K.S.; Cani, P.D.; et al. Expert consensus document: The International Scientific Association for Probiotics and Prebiotics (ISAPP) consensus statement on the definition and scope of prebiotics. Nat. Rev. Gastroenterol. Hepatol. 2017, 14, 491-502. [CrossRef]

30. Moorthy, M.; Chaiyakunapruk, N.; Jacob, S.A.; Palanisamy, U.D. Prebiotic potential of polyphenols, its effect on gut microbiota and anthropometric/clinical markers: A systematic review of randomised controlled trials. Trends Food Sci. Technol. 2020, 99, 634-649. [CrossRef]

31. Liberati, A.; Altman, D.G.; Tetzlaff, J.; Mulrow, C.; Gotzsche, P.C.; Ioannidis, J.P.; Clarke, M.; Devereaux, P.J.; Kleijnen, J.; Moher, D. The PRISMA statement for reporting systematic reviews and meta-analyses of studies that evaluate health care interventions: Explanation and elaboration. J. Clin. Epidemiol. 2009, 62, e1-e34. [CrossRef]

32. Hooijmans, C.R.; Rovers, M.M.; De Vries, R.B.; Leenaars, M.; Ritskes-Hoitinga, M.; Langendam, M.W. SYRCLE's risk of bias tool for animal studies. BMC Med. Res. Methodol. 2014, 14, 43. [CrossRef] [PubMed]

33. Hooijmans, C.R.; Leenaars, M.; Ritskes-Hoitinga, M. A Gold Standard Publication Checklist to Improve the Quality of Animal Studies, to Fully Integrate the Three Rs, and to Make Systematic Reviews More Feasible. Altern. Lab. Anim. 2010, 38, 167-182. [CrossRef] [PubMed]

34. Bule, M.H.; Abdurahman, A.; Nikfar, S.; Abdollahi, M.; Amini, M. Antidiabetic effect of quercetin: A systematic review and meta-analysis of animal studies. Food Chem. Toxicol. 2019, 125, 494-502. [CrossRef] [PubMed]

35. López, P.; Sánchez, M.; Perez-Cruz, C.; Velázquez-Villegas, L.A.; Syeda, T.; Aguilar-López, M.; Rocha-Viggiano, A.K.; Del Carmen Silva-Lucero, M.; Torre-Villalvazo, I.; Noriega, L.G. Long-Term Genistein Consumption Modifies Gut Microbiota, Improving Glucose Metabolism, Metabolic Endotoxemia, and Cognitive Function in Mice Fed a High-Fat Diet. Mol. Nutr. Food Res. 2018, 62, e1800313. [CrossRef] [PubMed]

36. Guo, X.; Tang, R.; Yang, S.; Lu, Y.; Luo, J.; Liu, Z. Rutin and Its Combination With Inulin Attenuate Gut Dysbiosis, the Inflammatory Status and Endoplasmic Reticulum Stress in Paneth Cells of Obese Mice Induced by High-Fat Diet. Front. Microbiol. 2018, 9, 2651. [CrossRef]

37. Masumoto, S.; Terao, A.; Yamamoto, Y.; Mukai, T.; Miura, T.; Shoji, T. Non-absorbable apple procyanidins prevent obesity associated with gut microbial and metabolomic changes. Sci. Rep. 2016, 6, 31208. [CrossRef]

38. Tung, Y.-C.; Lin, Y.-H.; Chen, H.-J.; Chou, S.-C.; Cheng, A.-C.; Nagabhushanam, K.; Ho, C.-T.; Pan, M.-H. Piceatannol Exerts Anti-Obesity Effects in C57BL/6 Mice through Modulating Adipogenic Proteins and Gut Microbiota. Molecules 2016, 21, 1419. [CrossRef]

39. Porras, D.; Nistal, E.; Martínez-Florez, S.; Pisonero-Vaquero, S.; Olcoz, J.L.; Jover, R.; González-Gallego, J.; García-Mediavilla, M.V.; Sánchez-Campos, S. Protective effect of quercetin on high-fat diet-induced non-alcoholic fatty liver disease in mice is mediated by modulating intestinal microbiota imbalance and related gut-liver axis activation. Free. Radic. Biol. Med. 2017, 102, 188-202. [CrossRef]

40. Brandt, N.; Kotowska, D.; Kristensen, C.M.; Olesen, J.; Lützhøft, D.O.; Halling, J.F.; Hansen, M.; Al-Soud, W.A.; Hansen, L.; Kiilerich, P.; et al. The impact of exercise training and resveratrol supplementation on gut microbiota composition in high-fat diet fed mice. Physiol. Rep. 2018, 6, e13881. [CrossRef]

41. Guo, J.; Han, X.; Zhan, J.; You, Y.; Huang, W. Vanillin Alleviates High Fat Diet-Induced Obesity and Improves the Gut Microbiota Composition. Front. Microbiol. 2018, 9, 2733. [CrossRef]

42. Cremonini, E.; Daveri, E.; Mastaloudis, A.; Adamo, A.M.; Mills, D.; Kalanetra, K.; Hester, S.N.; Wood, S.M.; Fraga, C.G.; Oteiza, P.I. Anthocyanins protect the gastrointestinal tract from high fat diet-induced alterations in redox signaling, barrier integrity and dysbiosis. Redox Biol. 2019, 26, 101269. [CrossRef] [PubMed]

43. Daveri, E.; Cremonini, E.; Mastaloudis, A.; Hester, S.N.; Wood, S.M.; Waterhouse, A.L.; Anderson, M.; Fraga, C.G.; Oteiza, P.I. Cyanidin and delphinidin modulate inflammation and altered redox signaling improving insulin resistance in high fat-fed mice. Redox Biol. 2018, 18, 16-24. [CrossRef] [PubMed]

44. Campbell, C.L.; Yu, R.; Li, F.; Zhou, Q.; Chen, D.; Qi, C.; Yin, Y.; Sun, J. Modulation of fat metabolism and gut microbiota by resveratrol on high-fat diet-induced obese mice. Diabetes Metab. Syndr. Obes. Targets Ther. 2019, 12, 97-107. [CrossRef] [PubMed]

45. Zheng, S.; Huang, K.; Zhao, C.; Xu, W.; Sheng, Y.; Luo, Y.; He, X. Procyanidin attenuates weight gain and modifies the gut microbiota in high fat diet induced obese mice. J. Funct. Foods 2018, 49, 362-368. [CrossRef]

46. Xie, M.; Chen, G.; Wan, P.; Dai, Z.; Zeng, X.; Sun, Y. Effects of Dicaffeoylquinic Acids from Ilex kudingcha on Lipid Metabolism and Intestinal Microbiota in High-Fat-Diet-Fed Mice. J. Agric. Food Chem. 2018, 67, 171-183. [CrossRef]

47. Sung, M.M.; Kim, T.; Denou, E.; Soltys, C.-L.M.; Hamza, S.M.; Byrne, N.J.; Masson, G.; Park, H.; Wishart, D.S.; Madsen, K.L.; et al. Improved Glucose Homeostasis in Obese Mice Treated With Resveratrol Is Associated With Alterations in the Gut Microbiome. Diabetes 2016, 66, 418-425. [CrossRef]

48. Liu, Z.; Wang, N.; Ma, Y.; Wen, D. Hydroxytyrosol Improves Obesity and Insulin Resistance by Modulating Gut Microbiota in High-Fat Diet-Induced Obese Mice. Front. Microbiol. 2019, 10, 390. [CrossRef]

49. Ushiroda, C.; Naito, Y.; Takagi, T.; Uchiyama, K.; Mizushima, K.; Higashimura, Y.; Yasukawa, Z.; Okubo, T.; Inoue, R.; Honda, A.; et al. Green tea polyphenol (epigallocatechin-3-gallate) improves gut dysbiosis and serum bile acids dysregulation in high-fat diet-fed mice. J. Clin. Biochem. Nutr. 2019, 65, 34-46. [CrossRef] 
50. Sheng, L.; Jena, P.K.; Liu, H.X.; Hu, Y.; Nagar, N.; Bronner, D.N.; Settles, M.L.; Bäumler, A.J.; Yvonne Wan, Y.-J. Obesity treatment by epigallocatechin-3-gallate-Regulated bile acid signaling and its enriched Akkermansia muciniphila. FASEB J. 2018, 32, 6371-6384. [CrossRef]

51. Liu, W.; Zhao, S.; Wang, J.; Shi, J.; Sun, Y.; Wang, W.; Ning, G.; Hong, J.; Liu, R. Grape seed proanthocyanidin extract ameliorates inflammation and adiposity by modulating gut microbiota in high-fat diet mice. Mol. Nutr. Food Res. 2017, 61, 1601082. [CrossRef]

52. Wang, Z.; Lam, K.; Hu, J.; Ge, S.; Zhou, A.; Zheng, B.; Zeng, S.-X.; Lin, S. Chlorogenic acid alleviates obesity and modulates gut microbiota in high-fat-fed mice. Food Sci. Nutr. 2019, 7, 579-588. [CrossRef] [PubMed]

53. Liao, W.; Yin, X.; Li, Q.; Zhang, H.; Liu, Z.; Zheng, X.; Zheng, L.; Feng, X. Resveratrol-Induced White Adipose Tissue Browning in Obese Mice by Remodeling Fecal Microbiota. Molecules 2018, 23, 3356. [CrossRef] [PubMed]

54. Zhou, M.; Zhang, Q.; Ma, W.; Tian, F.; Shen, H.; Zhou, M. A combination of quercetin and resveratrol reduces obesity in high-fat diet-fed rats by modulation of gut microbiota. Food Funct. 2017, 8, 4644-4656. [CrossRef] [PubMed]

55. Yang, C.; Deng, Q.; Xu, J.; Wang, X.; Hu, C.; Tang, H.; Huang, F.-H. Sinapic acid and resveratrol alleviate oxidative stress with modulation of gut microbiota in high-fat diet-fed rats. Food Res. Int. 2019, 116, 1202-1211. [CrossRef]

56. Luo, Q.; Cheng, D.; Huang, C.; Li, Y.; Lao, C.; Xia, Y.; Liu, W.; Gong, X.; Hu, D.; Li, B.; et al. Improvement of Colonic Immune Function with Soy Isoflavones in High-Fat Diet-Induced Obese Rats. Molecules 2019, 24, 1139. [CrossRef]

57. Bai, Y.; Wang, S.; Wang, X.; Weng, Y.; Fan, X.; Sheng, H.; Zhu, X.; Lou, L.; Zhang, F. The flavonoid-rich Quzhou Fructus Aurantii extract modulates gut microbiota and prevents obesity in high-fat diet-fed mice. Nutr. Diabetes 2019, 9, 1-11. [CrossRef]

58. Li, C.; Lin, K.; Li, K.; Deng, X.; Li, C. Reshaped fecal gut microbiota composition by the intake of high molecular weight persimmon tannin in normal and high-cholesterol diet-fed rats. Food Funct. 2017, 9, 541-551. [CrossRef]

59. Lee, H.; Kim, J.; An, J.; Lee, S.; Kong, H.; Song, Y.; Choi, H.R.; Lee, S.J.; Chae, K.S.; Kwon, J.W.; et al. Amelioration of hyperglycemia by Rubus occidentalis (black raspberry) and increase in short-chain fatty acids producing bacteria. J. Funct. Foods 2019, 54, 433-439. [CrossRef]

60. Collins, B.; Hoffman, J.; Martinez, K.; Grace, M.; Lila, M.A.; Cockrell, C.; Nadimpalli, A.; Chang, E.; Chuang, C.-C.; Zhong, W.; et al. A polyphenol-rich fraction obtained from table grapes decreases adiposity, insulin resistance and markers of inflammation and impacts gut microbiota in high-fat-fed mice. J. Nutr. Biochem. 2016, 31, 150-165. [CrossRef]

61. Guo, J.; Han, X.; Tan, H.; Huang, W.; You, Y.; Zhan, J. Blueberry Extract Improves Obesity through Regulation of the Gut Microbiota and Bile Acids via Pathways Involving FXR and TGR5. iScience 2019, 19, 676-690. [CrossRef]

62. Cheng, D.M.; Roopchand, D.E.; Poulev, A.; Kuhn, P.; Armas, I.; Johnson, W.D.; Oren, A.; Ribnicky, D.; Zelzion, E.; Bhattacharya, D.; et al. High phenolics Rutgers Scarlet Lettuce improves glucose metabolism in high fat diet-induced obese mice. Mol. Nutr. Food Res. 2016, 60, 2367-2378. [CrossRef] [PubMed]

63. Liu, Z.; Chen, Z.; Guo, H.; He, D.; Zhao, H.; Wang, Z.; Zhang, W.; Liao, L.; Zhang, C.; Ni, L. The modulatory effect of infusions of green tea, oolong tea, and black tea on gut microbiota in high-fat-induced obese mice. Food Funct. 2016, 7, 4869-4879. [CrossRef] [PubMed]

64. Griffin, L.E.; Witrick, K.A.; Klotz, C.; Dorenkott, M.R.; Goodrich, K.M.; Fundaro, G.; McMillan, R.P.; Hulver, M.W.; Ponder, M.A.; Neilson, A.P. Alterations to metabolically active bacteria in the mucosa of the small intestine predict anti-obesity and anti-diabetic activities of grape seed extract in mice. Food Funct. 2017, 8, 3510-3522. [CrossRef] [PubMed]

65. Xu, Y.-J.; Li, J.; Su, Q.; Liu, Y. Sinapine reduces non-alcoholic fatty liver disease in mice by modulating the composition of the gut microbiota. Food Funct. 2019, 10, 3637-3649. [CrossRef]

66. Ma, H.; Zhang, B.; Hu, Y.; Wang, J.; Liu, J.-M.; Qin, R.; Lv, S.; Wang, S. Correlation Analysis of Intestinal Redox State with the Gut Microbiota Reveals the Positive Intervention of Tea Polyphenols on Hyperlipidemia in High Fat Diet Fed Mice. J. Agric. Food Chem. 2019, 67, 7325-7335. [CrossRef] [PubMed]

67. Van Hul, M.; Geurts, L.; Plovier, H.; Druart, C.; Everard, A.; Ståhlman, M.; Rhimi, M.; Chira, K.; Teissedre, P.-L.; Delzenne, N.M.; et al. Reduced obesity, diabetes, and steatosis upon cinnamon and grape pomace are associated with changes in gut microbiota and markers of gut barrier. Am. J. Physiol. 2018, 314, E334. [CrossRef] [PubMed]

68. Anhê, F.F.; Roy, D.; Pilon, G.; Dudonné, S.; Matamoros, S.; Varin, T.V.; Garofalo, C.; Moine, Q.; Desjardins, Y.; Levy, E.; et al. A polyphenol-rich cranberry extract protects from diet-induced obesity, insulin resistance and intestinal inflammation in association with increased Akkermansia spp. population in the gut microbiota of mice. Gut 2015, 64, 872-883. [CrossRef]

69. Anhê, F.F.; Nachbar, R.T.; Varin, T.V.; Vilela, V.R.; Stephanie, D.; Pilon, G.; Fournier, M.; Lecours, M.-A.; Desjardins, Y.; Roy, D.; et al. A polyphenol-rich cranberry extract reverses insulin resistance and hepatic steatosis independently of body weight loss. Mol. Metab. 2017, 6, 1563-1573. [CrossRef]

70. Anhê, F.F.; Varin, T.V.; Le Barz, M.; Pilon, G.; Dudonné, S.; Trottier, J.; St-Pierre, P.; Harris, C.S.; Lucas, M.; Lemire, M.; et al. Arctic berry extracts target the gut-liver axis to alleviate metabolic endotoxaemia, insulin resistance and hepatic steatosis in diet-induced obese mice. Diabetologia 2018, 61, 919-931. [CrossRef]

71. Chen, G.; Xie, M.; Dai, Z.; Wan, P.; Ye, H.; Zeng, X.; Sun, Y. Kudingcha and Fuzhuan Brick Tea Prevent Obesity and Modulate Gut Microbiota in High-Fat Diet Fed Mice. Mol. Nutr. Food Res. 2018, 62, e1700485. [CrossRef]

72. Xu, J.; Liu, T.; Li, Y.; Liu, W.; Ding, Z.; Ma, H.; Seeram, N.P.; Mu, Y.; Huang, X.; Li, L. Jamun (Eugenia jambolana Lam.) Fruit Extract Prevents Obesity by Modulating the Gut Microbiome in High-Fat-Diet-Fed Mice. Mol. Nutr. Food Res. 2019, 63, e1801307. [CrossRef] [PubMed] 
73. Dey, P.; Sasaki, G.Y.; Wei, P.; Li, J.; Wang, L.; Zhu, J.; McTigue, D.; Yu, Z.; Bruno, R.S. Green tea extract prevents obesity in male mice by alleviating gut dysbiosis in association with improved intestinal barrier function that limits endotoxin translocation and adipose inflammation. J. Nutr. Biochem. 2019, 67, 78-89. [CrossRef] [PubMed]

74. Wu, S.; Hu, R.; Nakano, H.; Chen, K.; Liu, M.; He, X.; Zhang, H.; He, J.; Hou, D.-X. Modulation of Gut Microbiota by Lonicera caerulea L. Berry Polyphenols in a Mouse Model of Fatty Liver Induced by High Fat Diet. Molecules 2018, 23, 3213. [CrossRef]

75. Liu, M.; Tan, J.; He, Z.; He, X.; Hou, D.-X.; He, J.; Wu, S. Inhibitory effect of blue honeysuckle extract on high-fat-diet-induced fatty liver in mice. Anim. Nutr. 2018, 4, 288-293. [CrossRef] [PubMed]

76. Wu, C.; Tian, Y.; Yu, J.; Zhang, R.; Zhang, X.; Guo, P. The pandanus tectorius fruit extract (PTF) modulates the gut microbiota and exerts anti-hyperlipidaemic effects. Phytomedicine 2019, 58, 152863. [CrossRef] [PubMed]

77. Vezza, T.; Rodríguez-Nogales, A.; Algieri, F.; Garrido-Mesa, J.; Romero, M.; Sánchez, M.; Toral, M.; Martín-García, B.; GómezCaravaca, A.M.; Arráez-Román, D.; et al. The metabolic and vascular protective effects of olive (Olea europaea L.) leaf extract in diet-induced obesity in mice are related to the amelioration of gut microbiota dysbiosis and to its immunomodulatory properties. Pharmacol. Res. 2019, 150, 104487. [CrossRef]

78. Henning, S.M.; Yang, J.; Hsu, M.; Lee, R.-P.; Grojean, E.M.; Ly, A.; Tseng, C.-H.; Heber, D.; Li, Z. Decaffeinated green and black tea polyphenols decrease weight gain and alter microbiome populations and function in diet-induced obese mice. Eur. J. Nutr. 2018, 57, 2759-2769. [CrossRef]

79. Zhao, R.; Long, X.; Yang, J.; Du, L.; Zhang, X.; Li, J.; Hou, C. Pomegranate peel polyphenols reduce chronic low-grade inflammatory responses by modulating gut microbiota and decreasing colonic tissue damage in rats fed a high-fat diet. Food Funct. 2019, 10, 8273-8285. [CrossRef]

80. Lee, S.; I Keirsey, K.; Kirkland, R.; Grunewald, Z.I.; Fischer, J.G.; De La Serre, C.B. Blueberry Supplementation Influences the Gut Microbiota, Inflammation, and Insulin Resistance in High-Fat-Diet-Fed Rats. J. Nutr. 2018, 148, 209-219. [CrossRef]

81. Xu, H.; Zhao, C.; Li, Y.; Liu, R.; Ao, M.; Li, F.; Yao, Y.; Tao, Z.; Yu, L.-J. The ameliorative effect of the Pyracantha fortuneana (Maxim.) H. L. Li extract on intestinal barrier dysfunction through modulating glycolipid digestion and gut microbiota in high fat diet-fed rats. Food Funct. 2019, 10, 6517-6532. [CrossRef]

82. Ding, Y.; Song, Z.; Li, H.; Chang, L.; Pan, T.; Gu, X.; He, X.; Fan, Z. Honokiol Ameliorates High-Fat-Diet-Induced Obesity of Different Sexes of Mice by Modulating the Composition of the Gut Microbiota. Front. Immunol. 2019, 10. [CrossRef]

83. Liao, Z.-L.; Zeng, B.-H.; Wang, W.; Li, G.-H.; Wu, F.; Wang, L.; Zhong, Q.-P.; Wei, H.; Fang, X. Impact of the Consumption of Tea Polyphenols on Early Atherosclerotic Lesion Formation and Intestinal Bifidobacteria in High-Fat-Fed ApoE-/ - Mice. Front. Nutr. 2016, 3. [CrossRef] [PubMed]

84. Weisberg, S.P.; McCann, D.; Desai, M.; Rosenbaum, M.; Leibel, R.L.; Ferrante, A.W. Obesity is associated with macrophage accumulation in adipose tissue. J. Clin. Investig. 2003, 112, 1796-1808. [CrossRef]

85. Xu, H.; Barnes, G.T.; Yang, Q.; Tan, G.; Yang, D.; Chou, C.J.; Sole, J.; Nichols, A.; Ross, J.S.; Tartaglia, L.A.; et al. Chronic inflammation in fat plays a crucial role in the development of obesity-related insulin resistance. J. Clin. Investig. 2003, 112, 1821-1830. [CrossRef] [PubMed]

86. Wu, H.; Ballantyne, C.M. Metabolic inflammation and insulin resistance in obesity. Circ. Res. 2020, 126, 1549-1564. [CrossRef] [PubMed]

87. Shimobayashi, M.; Albert, V.; Woelnerhanssen, B.; Frei, I.C.; Weissenberger, D.; Meyer-Gerspach, A.C.; Clement, N.; Moes, S.; Colombi, M.; Meier, J.A.; et al. Insulin resistance causes inflammation in adipose tissue. J. Clin. Investig. 2018, 128, 1538-1550. [CrossRef]

88. Lee, Y.S.; Li, P.; Huh, J.Y.; Hwang, I.J.; Lu, M.; Kim, J.I.; Ham, M.; Talukdar, S.; Chen, A.; Lu, W.J.; et al. Inflammation Is Necessary for Long-Term but Not Short-Term High-Fat Diet-Induced Insulin Resistance. Diabetes 2011, 60, 2474-2483. [CrossRef]

89. Cheng, H.S.; Goh, B.H.; Phang, S.C.W.; Amanullah, M.M.; Ton, S.H.; Palanisamy, U.D.; Kadir, K.A.; Tan, J.B.L. Pleiotrosepic ameliorative effects of ellagitannin geraniin against metabolic syndrome induced by high-fat diet in rats. Nutrition 2020, 79-80, 110973. [CrossRef]

90. Vernochet, C.; Mourier, A.; Bezy, O.; Macotela, Y.; Boucher, J.; Rardin, M.J.; An, D.; Lee, K.Y.; Ilkayeva, O.R.; Zingaretti, C.M.; et al. Adipose-Specific Deletion of TFAM Increases Mitochondrial Oxidation and Protects Mice against Obesity and Insulin Resistance. Cell Metab. 2012, 16, 765-776. [CrossRef]

91. Yang, J.; Guo, Y.; Henning, S.; Chan, B.; Long, J.; Zhong, J.; Acin-Perez, R.; Petcherski, A.; Shirihai, O.; Heber, D.; et al. Ellagic Acid and Its Microbial Metabolite Urolithin a Alleviate Diet-induced Insulin Resistance in Mice (OR24-03-19). Curr. Dev. Nutr. 2019, 3 (Suppl. 1), nzz031-OR24. [CrossRef]

92. Perera, A.; Ton, S.H.; Moorthy, M.; Palanisamy, U.D. The insulin-sensitising properties of the ellagitannin geraniin and its metabolites from Nephelium lappaceum rind in 3T3-L1 cells. Int. J. Food Sci. Nutr. 2020, 2020, 940-953. [CrossRef] [PubMed]

93. Aryaeian, N.; Sedehi, S.K.; Arablou, T. Polyphenols and their effects on diabetes management: A review. Med. J. Islam. Repub. Iran. 2017, 31, 886-892. [CrossRef] [PubMed]

94. Williamson, G.; Sheedy, K. Effects of Polyphenols on Insulin Resistance. Nutrients 2020, 12, 3135. [CrossRef] [PubMed]

95. Sun, C.; Zhao, C.; Guven, E.C.; Paoli, P.; Simal-Gandara, J.; Ramkumar, K.M.; Wang, S.; Buleu, F.; Pah, A.; Turi, V.; et al. Dietary polyphenols as antidiabetic agents: Advances and opportunities. Food Front. 2020, 1, 18-44. [CrossRef] 
96. Kerimi, A.; Nyambe-Silavwe, H.; Gauer, J.S.; Tomás-Barberán, F.A.; Williamson, G. Pomegranate juice, but not an extract, confers a lower glycemic response on a high-glycemic index food: Randomized, crossover, controlled trials in healthy subjects. Am. J. Clin. Nutr. 2017, 106, 1384-1393. [CrossRef]

97. Adisakwattana, S.; Chantarasinlapin, P.; Thammarat, H.; Yibchok-Anun, S. A series of cinnamic acid derivatives and their inhibitory activity on intestinal $\alpha$-glucosidase. J. Enzym. Inhib. Med. Chem. 2009, 24, 1194-1200. [CrossRef]

98. Song, J.; Kwon, O.; Chen, S.; Daruwala, R.; Eck, P.; Park, J.B.; Levine, M. Flavonoid Inhibition of Sodium-dependent Vitamin C Transporter 1 (SVCT1) and Glucose Transporter Isoform 2 (GLUT2), Intestinal Transporters for Vitamin C and Glucose. J. Biol. Chem. 2002, 277, 15252-15260. [CrossRef]

99. Villa-Rodriguez, J.A.; Aydin, E.; Gauer, J.S.; Pyner, A.; Williamson, G.; Kerimi, A. Green and Chamomile Teas, but not Acarbose, Attenuate Glucose and Fructose Transport via Inhibition of GLUT2 and GLUT. Mol. Nutr. Food Res. 2017, 61. [CrossRef]

100. Houghton, M.J.; Kerimi, A.; Mouly, V.; Tumova, S.; Williamson, G. Gut microbiome catabolites as novel modulators of muscle cell glucose metabolism. FASEB J. 2018, 33, 1887-1898. [CrossRef]

101. Ueda-Wakagi, M.; Mukai, R.; Fuse, N.; Mizushina, Y.; Ashida, H. 3-O-Acyl-epicatechins Increase Glucose Uptake Activity and GLUT4 Translocation through Activation of PI3K Signaling in Skeletal Muscle Cells. Int. J. Mol. Sci. 2015, 16, 16288-16299. [CrossRef]

102. So, D.; Whelan, K.; Rossi, M.; Morrison, M.; Holtmann, G.J.; Kelly, J.T.; Shanahan, E.R.; Staudacher, H.M.; Campbell, K.L. Dietary fiber intervention on gut microbiota composition in healthy adults: A systematic review and meta-analysis. Am. J. Clin. Nutr. 2018, 107, 965-983. [CrossRef] [PubMed]

103. Li, H.; Li, T.; Beasley, D.E.; Heděnec, P.; Xiao, Z.; Zhang, S.; Li, J.; Lin, Q.; Li, X. Diet Diversity Is Associated with Beta but not Alpha Diversity of Pika Gut Microbiota. Front. Microbiol. 2016, 7, 1169. [CrossRef] [PubMed]

104. Castañer, O.; Goday, A.; Park, Y.-M.; Lee, S.; Magkos, F.; Shiow, S.-A.T.E.; Schröder, H. The Gut Microbiome Profile in Obesity: A Systematic Review. Int. J. Endocrinol. 2018, 2018, 1-9. [CrossRef]

105. De Wit, N.; Derrien, M.; Bosch-Vermeulen, H.; Oosterink, E.; Keshtkar, S.; Duval, C.; de Vogel-van den Bosch, J.; Kleerebezem, M.; Müller, M.; van der Meer, R. Saturated fat stimulates obesity and hepatic steatosis and affects gut microbiota composition by an enhanced overflow of dietary fat to the distal intestine. Am. J. Physiol. Gastrointest. Liver Physiol. 2012, 303, G589-G599. [CrossRef]

106. Hildebrandt, M.A.; Hoffmann, C.; Sherrill-Mix, S.A.; Keilbaugh, S.A.; Hamady, M.; Chen, Y.; Knight, R.; Ahima, R.S.; Bushman, F.; Wu, G.D. High-Fat Diet Determines the Composition of the Murine Gut Microbiome Independently of Obesity. Gastroenterology 2009, 137, 1716-1724.e2. [CrossRef] [PubMed]

107. Tims, S.; Derom, C.; Jonkers, D.; Vlietinck, R.; Saris, W.H.; Kleerebezem, M.; De Vos, W.M.; Zoetendal, E.G. Microbiota conservation and BMI signatures in adult monozygotic twins. ISME J. 2013, 7, 707-717. [CrossRef]

108. Patil, D.P.; Dhotre, D.P.; Chavan, S.G.; Sultan, A.; Jain, D.S.; Lanjekar, V.B.; Gangawani, J.; Shah, P.S.; Todkar, J.S.; Shah, S.; et al. Molecular analysis of gut microbiota in obesity among Indian individuals. J. Biosci. 2012, 37, 647-657. [CrossRef]

109. Jumpertz, R.; Le, D.S.; Turnbaugh, P.J.; Trinidad, C.; Bogardus, C.; Gordon, J.I.; Krakoff, J. Energy-balance studies reveal associations between gut microbes, caloric load, and nutrient absorption in humans. Am. J. Clin. Nutr. 2011, 94, 58-65. [CrossRef]

110. Sze, M.A.; Schloss, P.D. Looking for a Signal in the Noise: Revisiting Obesity and the Microbiome. $m$ Bio 2016, 7, e01018-16. [CrossRef]

111. Walters, W.A.; Xu, Z.; Knight, R. Meta-analyses of human gut microbes associated with obesity and IBD. FEBS Lett. 2014, 588, 4223-4233. [CrossRef]

112. Finucane, M.M.; Sharpton, T.J.; Laurent, T.J.; Pollard, K.S. A Taxonomic Signature of Obesity in the Microbiome? Getting to the Guts of the Matter. PLoS ONE 2014, 9, e84689. [CrossRef]

113. Magne, F.; Gotteland, M.; Gauthier, L.; Zazueta, A.; Pesoa, S.; Navarrete, P.; Balamurugan, R. The Firmicutes/Bacteroidetes Ratio: A Relevant Marker of Gut Dysbiosis in Obese Patients? Nutrients 2020, 12, 1474. [CrossRef] [PubMed]

114. Xu, Y.; Wang, N.; Tan, H.-Y.; Li, S.; Zhang, C.; Feng, Y. Function of Akkermansia muciniphila in Obesity: Interactions with Lipid Metabolism, Immune Response and Gut Systems. Front. Microbiol. 2020, 11, 219. [CrossRef] [PubMed]

115. Kim, S.; Lee, Y.; Kim, Y.; Seo, Y.; Lee, H.; Ha, J.; Lee, J.; Choi, Y.; Oh, H.; Yoon, Y. Prevents Fatty Liver Disease, Decreases Serum Triglycerides, and Maintains Gut Homeostasis. Appl. Environ. Microbiol. 2020, 86, e03004-19. [CrossRef] [PubMed]

116. Depommier, C.; Everard, A.; Druart, C.; Plovier, H.; Van Hul, M.; Vieira-Silva, S.; Falony, G.; Raes, J.; Maiter, D.; Delzenne, N.M.; et al. Supplementation with Akkermansia muciniphila in overweight and obese human volunteers: A proof-of-concept exploratory study. Nat. Med. 2019, 25, 1096-1103. [CrossRef] [PubMed]

117. Dahiya, D.K.; Renuka; Dangi, A.K.; Shandilya, U.K.; Puniya, A.K.; Shukla, P. Chapter 44-New-Generation Probiotics: Perspectives and Applications. In Microbiome and Metabolome in Diagnosis, Therapy, and other Strategic Applications; Faintuch, J., Faintuch, S., Eds.; Academic Press: Cambridge, MA, USA, 2019; pp. 417-424.

118. Sears, C.L.; Franco, A.A.; Wu, S. CHAPTER 28-Bacteroides fragilis toxins. In The Comprehensive Sourcebook of Bacterial Protein Toxins, 3rd ed.; Alouf, J.E., Popoff, M.R., Eds.; Academic Press: London, UK, 2006; pp. 535-546.

119. Benítez-Páez, A.; Del Pugar, E.M.G.; López-Almela, I.; Moya-Pérez, Á.; Codoñer-Franch, P.; Sanz, Y.; Jenq, R. Depletion of Blautia Species in the Microbiota of Obese Children Relates to Intestinal Inflammation and Metabolic Phenotype Worsening. mSystems 2020, 5. [CrossRef] 
120. Ozato, N.; Saito, S.; Yamaguchi, T.; Katashima, M.; Tokuda, I.; Sawada, K.; Katsuragi, Y.; Kakuta, M.; Imoto, S.; Ihara, K.; et al. Blautia genus associated with visceral fat accumulation in adults 20-76 years of age. NPJ Biofilms Microbiomes 2019, 5, 1-9. [CrossRef]

121. Karlsson, F.H.; Tremaroli, V.; Nookaew, I.; Bergström, G.; Behre, C.J.; Fagerberg, B.; Nielsen, J.; Bäckhed, F. Gut metagenome in European women with normal, impaired and diabetic glucose control. Nature 2013, 498, 99-103. [CrossRef]

122. Smecuol, E.; Hwang, H.J.; Sugai, E.; Corso, L.; Cherñavsky, A.C.; Bellavite, F.P.; González, A.; Vodánovich, F.; Moreno, M.L.; Vázquez, H.; et al. Exploratory, Randomized, Double-blind, Placebo-controlled Study on the Effects of Bifidobacterium infantis Natren Life Start Strain Super Strain in Active Celiac Disease. J. Clin. Gastroenterol. 2013, 47, 139-147. [CrossRef]

123. Agrawal, A.; Houghton, L.A.; Morris, J.; Reilly, B.; Guyonnet, D.; Feuillerat, N.G.; Schlumberger, A.; Jakob, S.; Whorwell, P.J. Clinical trial: The effects of a fermented milk product containingBifidobacterium lactisDN-173 010 on abdominal distension and gastrointestinal transit in irritable bowel syndrome with constipation. Aliment. Pharmacol. Ther. 2009, 29, 104-114. [CrossRef]

124. Kadooka, Y.; Sato, M.; Imaizumi, K.; Ogawa, A.; Ikuyama, K.; Akai, Y.; Okano, M.; Kagoshima, M.; Tsuchida, T. Regulation of abdominal adiposity by probiotics (Lactobacillus gasseri SBT2055) in adults with obese tendencies in a randomized controlled trial. Eur. J. Clin. Nutr. 2010, 64, 636-643. [CrossRef] [PubMed]

125. Chung, H.; Yu, J.G.; Lee, I.; Liu, M.; Shen, Y.; Sharma, S.P.; Jamal, M.A.H.M.; Yoo, J.; Kim, H.; Hong, S. Intestinal removal of free fatty acids from hosts by Lactobacilli for the treatment of obesity. FEBS Open Biol. 2016, 6, 64-76. [CrossRef] [PubMed]

126. Million, M.; Angelakis, E.; Paul, M.; Armougom, F.; Leibovici, L.; Raoult, D. Comparative meta-analysis of the effect of Lactobacillus species on weight gain in humans and animals. Microb. Pathog. 2012, 53, 100-108. [CrossRef] [PubMed]

127. Shao, L.; Ling, Z.; Chen, D.; Liu, Y.; Yang, F.; Li, L. Disorganized Gut Microbiome Contributed to Liver Cirrhosis Progression: A Meta-Omics-Based Study. Front. Microbiol. 2018, 9, 3166. [CrossRef] [PubMed]

128. Moschen, A.R.; Gerner, R.R.; Wang, J.; Klepsch, V.; Adolph, T.E.; Reider, S.J.; Hackl, H.; Pfister, A.; Schilling, J.; Moser, P.L.; et al. Lipocalin 2 Protects from Inflammation and Tumorigenesis Associated with Gut Microbiota Alterations. Cell Host Microbe 2016, 19, 455-469. [CrossRef] [PubMed]

129. Rowan, F.; Docherty, N.G.; Murphy, M.; Murphy, B.; Coffey, J.C.; O’Connell, P.R. Desulfovibrio Bacterial Species Are Increased in Ulcerative Colitis. Dis. Colon Rectum 2010, 53, 1530-1536. [CrossRef]

130. Cani, P.D.; Amar, J.; Iglesias, M.A.; Poggi, M.; Knauf, C.; Bastelica, D.; Neyrinck, A.M.; Fava, F.; Tuohy, K.M.; Chabo, C.; et al. Metabolic Endotoxemia Initiates Obesity and Insulin Resistance. Diabetes 2007, 56, 1761-1772. [CrossRef]

131. Cani, P.D.; Bibiloni, R.; Knauf, C.; Waget, A.; Neyrinck, A.M.; Delzenne, N.M.; Burcelin, R. Changes in Gut Microbiota Control Metabolic Endotoxemia-Induced Inflammation in High-Fat Diet-Induced Obesity and Diabetes in Mice. Diabetes 2008, 57, 1470-1481. [CrossRef]

132. Vrieze, A.; Out, C.; Fuentes, S.; Jonker, L.; Reuling, I.; Kootte, R.S.; Van Nood, E.; Holleman, F.; Knaapen, M.; Romijn, J.A.; et al. Impact of oral vancomycin on gut microbiota, bile acid metabolism, and insulin sensitivity. J. Hepatol. 2014, 60, 824-831. [CrossRef]

133. Gao, Z.; Yin, J.; Zhang, J.; Ward, R.E.; Martin, R.J.; Lefevre, M.; Cefalu, W.T.; Ye, J. Butyrate Improves Insulin Sensitivity and Increases Energy Expenditure in Mice. Diabetes 2009, 58, 1509-1517. [CrossRef] 